\title{
An Insulating Constriction Dielectrophoresis Based Microfluidic Device for Protein Biomolecules Concentration
}

\author{
Peng Zhang
}

Follow this and additional works at: https://researchrepository.wvu.edu/etd

\section{Recommended Citation}

Zhang, Peng, "An Insulating Constriction Dielectrophoresis Based Microfluidic Device for Protein Biomolecules Concentration" (2017). Graduate Theses, Dissertations, and Problem Reports. 7041. https://researchrepository.wvu.edu/etd/7041

This Thesis is protected by copyright and/or related rights. It has been brought to you by the The Research Repository @ WVU with permission from the rights-holder(s). You are free to use this Thesis in any way that is permitted by the copyright and related rights legislation that applies to your use. For other uses you must obtain permission from the rights-holder(s) directly, unless additional rights are indicated by a Creative Commons license in the record and/ or on the work itself. This Thesis has been accepted for inclusion in WVU Graduate Theses, Dissertations, and Problem Reports collection by an authorized administrator of The Research Repository @ WVU. For more information, please contact researchrepository@mail.wvu.edu. 


\title{
AN INSULATING CONSTRICTION DIELECTROPHORESIS BASED MICROFLUIDIC DEVICE FOR PROTEIN BIOMOLECULES CONCENTRATION
}

\author{
Peng Zhang \\ Thesis submitted to the \\ Benjamin M. Statler College of Engineering and Mineral Resources \\ at West Virginia University \\ in partial fulfillment of the requirements \\ for the degree of \\ Master of Science \\ in \\ Electrical Engineering \\ Yuxin Liu, Ph.D., Chairperson and Research Advisor \\ Mark Jerabek, Ph.D. \\ Jeremy Dawson, Ph.D. \\ Lane Department of Computer Science and Electrical Engineering \\ Morgantown, West Virginia \\ November 2017
}

Keywords: insulator-based dielectrophoresis, constricted channel, electrokinetics, protein concentration, microfluidics, molecular combing

Copyright 2017 Peng Zhang 


\title{
ABSTRACT \\ An insulating constriction dielectrophoresis based microfluidic device for protein biomolecules concentration
}

\author{
Peng Zhang
}

Sample enrichment or molecules concentration has been considered as an essential step for sample processing and biomarker detection recently in many applications involving miniaturized devices aiming at biosensing and bioanalysis, including the development of specialized tests for the detection of specific proteins and antibodies in human blood with the help of microfluidic and lab-on-a-chip devices. Among all the means involved to achieve this aim, dielectrophoresis (DEP) is increasingly employed in molecule manipulation and concentration because it is nondestructive and ensures high efficiency. However, there are still constraints on implementing the required functions using the dielectrophoresis technique in the devised micro-scale structures with high throughput, as well as the technical challenge in integration of sensors and concentration units for low-abundance molecular detection.

In the present work, we demonstrated a methodology to achieve protein concentration utilizing the combination effects of electrokinetics and low frequency insulator-based dielectrophoresis (iDEP) generated within a microfluidic device, in which a submicron constricted channel was fabricated using DNA molecular combing and replica molding. This fabrication technique, avoids using e-beam lithography or other complicated nanochannel fabrication methods, provides an easy and low cost approach with the flexibility in controlling channel dimensions to create highly constricted channels embedded in a microfluidic device. With theoretical analysis and experiments, we demonstrated that albumin-fluorescein isothiocyanate conjugate (FITC-BSA) protein molecules can be significantly concentrated to form an arc-shaped band near the constricted channel under the effects of negative dielectrophoretic force and DC electrokinetic forces within 2-3 minutes. It was also observed that the amplitudes of the applied DC and AC electric fields, AC frequencies, as well as suspending medium conductivities had strong effects on the concentration responses of the FITC-BSA molecules, including the concentrated area and position, intensities of the focused molecules, and concentration speed.

Our method demonstrated in the thesis provides a simple and flexible approach for quickly concentrating protein molecules by controlling the applied electric field parameters. The iDEP device reported in this thesis can be used as a stand-alone sensor or worked as a preconcentration module integrated with biosensors for protein biomarker detections. Furthermore, low frequency dielectrophoresis provides practical uses for integrating the concentration module with a portable biosensing system. 


\section{Acknowledgements}

First of all, I would like to express my sincere gratitude to my adviser Dr. Yuxin Liu, whose continuous guidance, inspiration, and encouragement have been invaluable for my Master's thesis work. It is my extreme privilege to have been a graduate research assistant in her lab for the past two years. I am extremely lucky to have been given the chance of joining her group, and discover a whole new world in the field of microfluidics. I will always remember her as not only my advisor, but also my mentor of life.

I gratefully acknowledge the members of my committee: Dr. Yuxin Liu, and Dr. Jeremy Dawson and Dr. Mark Jerabek, for their valuable time and guidance of my research work as well as the valuable feedback on this thesis.

I would like to express my immense gratitude to my fellow lab mates: Jianbo Sun, and my former lab mate, Veronica. Their friendship helped me stay sane through these difficult years. Their support and care helped me overcome setbacks and stay focused on my graduate study. I greatly value their friendship and I deeply appreciate their belief in me.

Moreover, I would like to thank Dr. Kolin Brown, Dr. Weiqiang Ding, Mr. Harley Hart and the SRF staff for their great help with cleanroom-based microfabrication.

I would like to save the last word of acknowledgement for my family for all their love, support, and continuous encouragements throughout my years of study and through the process of researching and writing this thesis. This accomplishment would not have been possible without them. To them I dedicate this thesis. 


\section{Table of Contents}

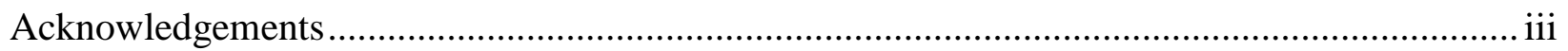

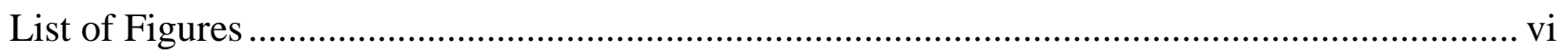

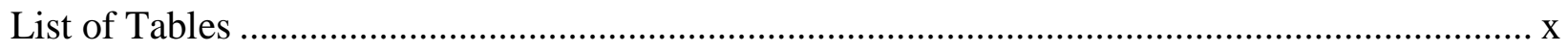

Chapter 1 Thesis Overview................................................................................................ 1

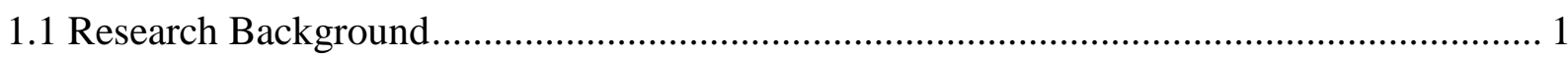

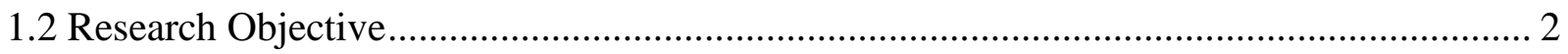

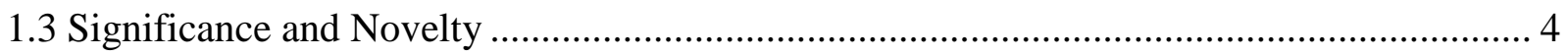

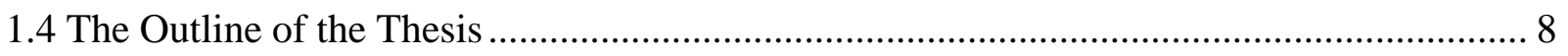

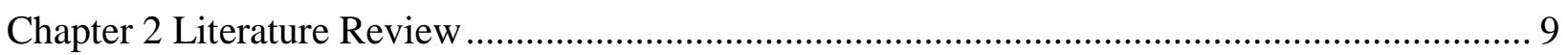

2.1 Dielectrophoresis-based Microfluidic Device................................................................ 9

2.2 State-of-Art Development of iDEP devices ............................................................... 12

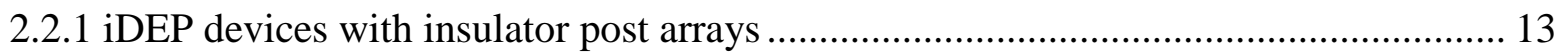

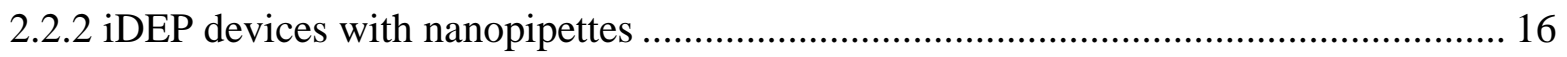

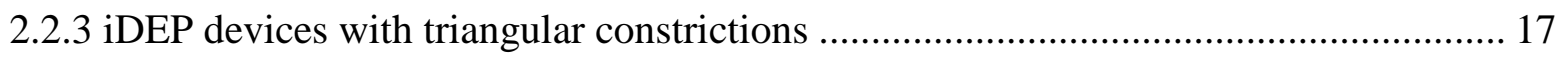

2.3 Chapter Summary ................................................................................................... 20

Chapter 3 Dielectrophoresis Theory and Principle of Protein Concentration ............................... 21

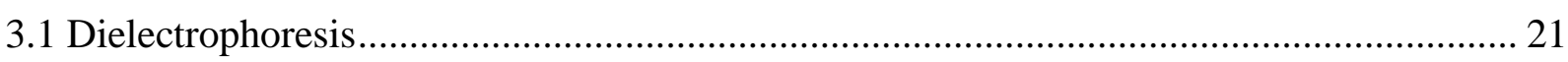

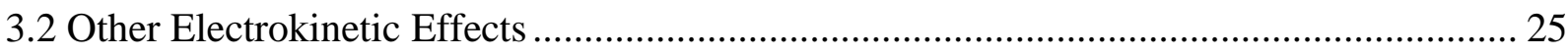

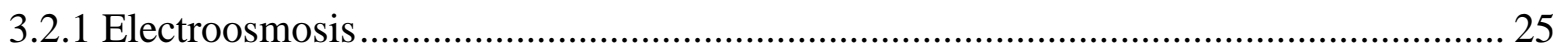

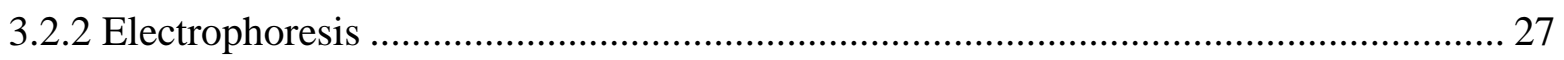

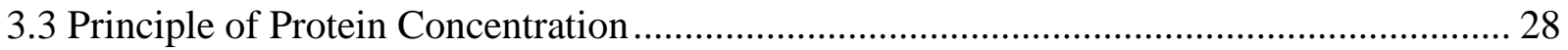

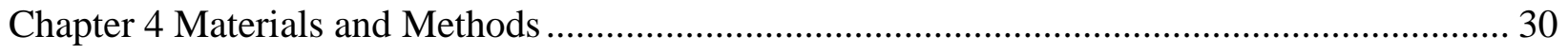

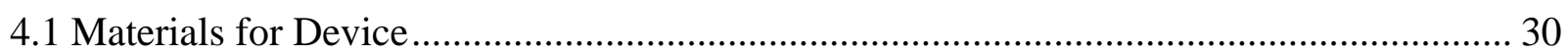

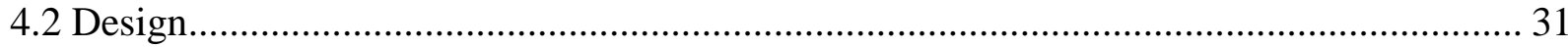

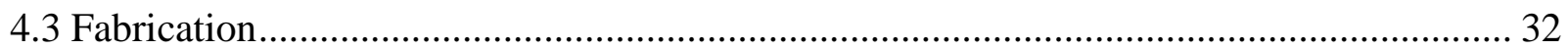

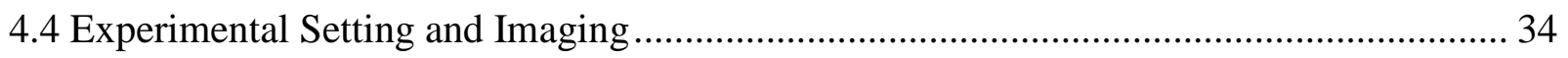

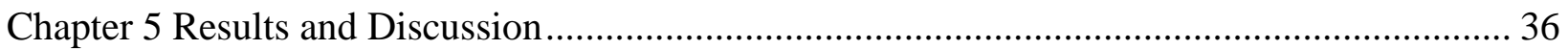

5.1 Fabrication of Constricted Channel within Microfluidic Device ......................................... 36

5.2 Effects of Electric Field Parameters .................................................................................. 38 


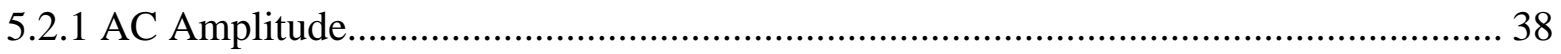

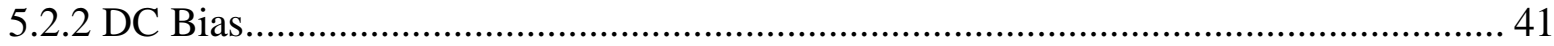

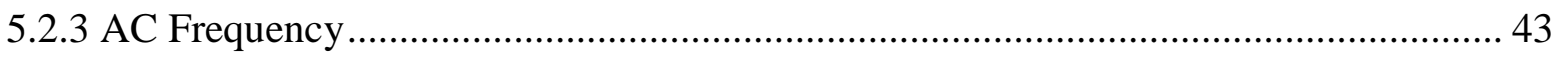

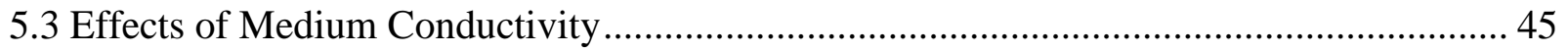

5.4 Measurement Sensitivity of Protein Concentration .................................................... 47

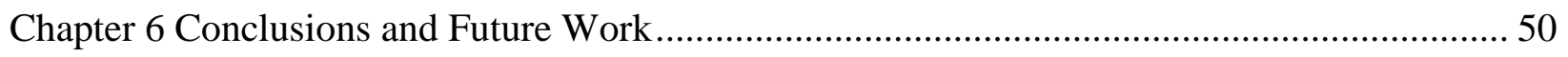

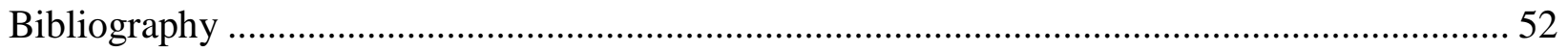




\section{List of Figures}

Figure 1-1. Fabrication and operation of the NEP device. (a) Schematic showing the fabrication of the NEP chip by DCI. (b) Left: schematic of an NEP chip covered by a PDMS lid with electrodes placed in reservoirs. Middle: optical micrograph of a cell in the left microchannel and positioned at the tip of the nanochannel using optical tweezers. The right microchannel contains gene or drugs. Right: SEM image of side view cut of a nanochannel. The nanochannel is $\sim 90 \mathrm{~nm}$ in diameter and $\sim 3 \mathrm{~mm}$ long [30]. 6

Figure 2-1. Examples of eDEP and iDEP structures. (a) eDEP design consisting of rectangular electrodes with trapping regions shown. (b) iDEP design consisting of circular insulating posts with trapping regions shown. 10

Figure 2-2. Shapes of eDEP device: (a) Micro tip electrode array. (b) and (c) Castellated electrode arrays with normal and shifted configurations. 11

Figure 2-3. Common insulating obstacle geometries reported in the literature. Red zones indicate where the particles experience the strongest dielectrophoretic effect.

Figure 2-4. Schematic of a microfluidic DEP trapping. (a) A metallic DEP trap made of microfabricated wire(s) on a substrate. The wire(s) may be either free-floating or connected to a voltage source. (b) An electrodeless DEP trap made of dielectric constrictions. The solid lines are electric field lines E. (c) A scanning electron micrograph of an electrodeless DEP device consisted of a constriction array etched in quartz. The constrictions are $1 \mu \mathrm{m}$ wide and $1.25 \mu \mathrm{m}$ deep. The whole chip measures $1 \times 1 \mathrm{~cm}$. The applied electric field direction $\mathrm{z}$ is shown by the double-headed arrow.

Figure 2-5. Schematic of the microfluidic device and a magnification of the microchannel containing insulating circular post arrays.

Figure 2-6. Schematic of the direct current insulator gradient dielectrophoresis device. An insulated sawtooth pattern is integrated with a tapered microfluidic channel to generate localized non-uniform electric field gradients of increasing strength from left to right. 15 
Figure 2-7. Fluorescence image recorded by using wide-field illumination $(5.2 \mathrm{~mW})$ of a nanopipette filled with $100 \mathrm{nM}$ Alexa-488 dye in PBS buffer...... 16

Figure 2-8. (a) Fluorescein-labeled single-stranded $1 \mathrm{~kb}$ DNA and double-stranded T5 DNA (103 kbp) trapped at a PDMS constriction array in 1x PCR buffer.. The constrictions are depicted by their triangular contours. The applied field was $200 \mathrm{Vpp} / \mathrm{cm}$ at $1 \mathrm{kHz}$ in $0.5 \mathrm{x}$ TBE buffer. (b) iDEP device for cell capture and enrichment. The constriction traps was fabricated in silicone elastomer using soft lithography sealed against a glass slide and have a $4 \mu \mathrm{m}$ opening and are 10 $\mu \mathrm{m}$ deep, with channel width ranging from 100-250 $\mu \mathrm{m}$ and various radius of curvature. The images on the right show the trapping of GFP-expressing E. Coli in a buffer with $50 \mathrm{mM}$ histidine, $250 \mathrm{mM}$ sucrose. The applied field was $300 \mathrm{Vpp} / \mathrm{cm}$ at $1 \mathrm{kHz}$.

Figure 2-9. Concept of nanoscale molecular trap and dam. (a) Positive dielectrophoresis (pDEP). (b) Negative dielectrophoresis (nDEP). (c) Molecular trap and dam via the combination of nDEP and a DC bias. 18

Figure 2-10. Protein trapping and damming with nanoscale eDEP. (a) Proteins (Alexa-488 streptavidins, $10 \mu \mathrm{g} / \mathrm{mL}$ ) loaded in the chip by capillary force trapping with pDEP. (b) 20 and (c) $40 \mathrm{~s}$ after $473 \mathrm{Vpp} / \mathrm{cm}$ ac field applied across the chip at $10 \mathrm{kHz}$. This field is focused $7 \times 10^{4}$-fold $\left(\sim 3.3 \times 10^{9} \mathrm{Vpp} / \mathrm{m}\right)$ over the applied field at the constrictions. All three constrictions show trapping of streptavidins, indicating PDEP is at work. (d) (f) Protein damming demonstrated by $\mathrm{nDEP}+\mathrm{DC}$ : ac field of $214 \mathrm{Vpp} / \mathrm{cm}$ is applied at $1 \mathrm{MHz}$ with $1.5 \mathrm{~V} / \mathrm{cm}$ DC bias (with positive potential at the right side of the channel). The dark zone at the center of the first constriction indicates NDEP is at work where streptavidins are repelled.

Figure 3-1. Schematic representation of a mechanism of DEP occurring under an inhomogeneous electric field by which a particle is transported (a) toward the higher electric field gradient (positive DEP) or (b) away from it (negative DEP). 23

Figure 3-2. (a): CM factor vs. frequency for specific particle conductivities. (b): CM factor vs. frequency for specific medium conductivities. 24

Figure 3-3. (a) Schematics of electrical double layer (EDL). The solid surface is negatively charged and the positive charges are electrostatically attracted to the interface, resulting in the Stern layer. (b) EOF profile in a microfluidic channel. Applied electric field direction: left to right. On the negatively charged wall (gray), positive counter ions are adsorbed, creating an 
immobile Stern layer. Fluid velocity is uniform throughout the cross section of the channel and drops to zero at the interface (no-slip). .26

Figure 3-4. Schematic diagram for protein concentration under dielectrophoresis and electrokinetics with an applied DC-biased AC electric field. 29

Figure 4-1. (a) Pattern of the PDMS mold of the designed microfluidic device. (b) Pattern of the electrodes for applying electric field.

Figure 4-2. Schematic fabrication process of a PDMS microfluidic device with constricted channels embedded. (a) Stretching DNA nanowire across suspending between two microridges. (b) Sputtered gold-coated DNA nanowires as templates for the constricted channels. (c) PDMS replica cast molding over the gold-coated stamps. (d) Microfluidic device with the embedded constricted channels after removing gold-coated DNA. 32

Figure 4-3. Schematic of the hypothetical process for the formation of DNA nanowires. 33

Figure 5-1. (a) Fluorescence image of YOYO-1 labeled DNA nanowires across suspending between two microridges. (b) Representative image shows gold-coated DNA nanowires connecting two microridges. (c) Representative image shows the constricted channel embedded within PDMS and connecting two microchannels after replica molding and gold etching. (d) An assembled microfluidic device for protein concentration. 37

Figure 5-2. Fluorescence images show FITC-BSA concentration results when an AC electric field $(1 \mathrm{kHz})$ was applied for 100 seconds with the condition of (a) $\mathrm{V}_{P P}=5 \mathrm{~V}, \mathrm{~V}_{D C}=0 \mathrm{~V}$, (b) $\mathrm{V}_{P P}=5 \mathrm{~V}, \mathrm{~V}_{D C}=1 \mathrm{~V}$, (c) $\mathrm{V}_{P P}=10 \mathrm{~V}, \mathrm{~V}_{D C}=1 \mathrm{~V}$, (d) $\mathrm{V}_{P P}=15 \mathrm{~V}, \mathrm{~V}_{D C}=1 \mathrm{~V}$, respectively. (e) Fluorescence intensity under different electric fields conditions. The $\mathrm{x}$-axis indicates the horizontal positions across the image plane from left end to right end, and the intensity values were measured along the direction of constricted channel at the middle vertical height for every condition, as illustrated by the flat dotted line shown in figure (c).. 39

Figure 5-3. Representative FITC-BSA concentration results under $\mathrm{AC}$ electric field $\left(\mathrm{V}_{P P}=15 \mathrm{~V}\right.$, frequency $=10 \mathrm{~Hz}$ ) with different DC biases. (a) (c) Fluorescence images show the results under AC electric field with $1 \mathrm{~V}$ DC bias after 10 seconds, 50 seconds and 100 seconds, respectively; (d) (f) Fluorescence images show the results under AC electric filed with 2V DC bias after 10 seconds, 50 seconds and 100 seconds, respectively; (g) Fluorescence intensities of the 
concentration over time under $\mathrm{AC}$ electric fields $\left(\mathrm{V}_{P P}=15 \mathrm{~V}\right)$ at frequencies of $10 \mathrm{~Hz}$ and $1 \mathrm{kHz}$ applied with $1 \mathrm{~V}$ and $2 \mathrm{~V}$ DC biases, respectively.

Figure 5-4. Representative FITC-BSA concentration results under different AC frequencies with the fixed $A C$ amplitude $\left(V_{P P}=15 V\right)$ and DC bias (2V). (a) (c) Fluorescence images show the results under $1 \mathrm{kHz}$ frequency after 10 seconds, 50 seconds and 100 seconds, respectively. (d) (f) Fluorescence images show the results under $10 \mathrm{~Hz}$ frequency after 10 seconds, 50 seconds and 100 seconds, respectively 44

Figure 5-5. FITC-BSA concentration results obtained under a combination of varying medium conductivities and $\mathrm{AC}$ frequencies with the fixed $\mathrm{AC}$ amplitude $\left(\mathrm{V}_{P P}=15 \mathrm{~V}\right)$ and $\mathrm{DC}$ bias $(2 \mathrm{~V})$. (a) Results obtained under $0.05 \times, 0.1 \times$, and $0.2 \times$ PBS; (b) Results obtained under $0.5 \times$ and $1 \times$ PBS.

Figure 5-6. Representative FITC-BSA concentration results under the same electric field condition $\left(\mathrm{V}_{P P}=15 \mathrm{~V}\right.$, frequency $\left.=10 \mathrm{~Hz}, \mathrm{~V}_{D C}=2 \mathrm{~V}\right)$ with different initial solution concentrations. (a) (f) Fluorescence images show the results after the electric field applied for 120 seconds using an initial solution concentration of $100 \mathrm{ng} / \mathrm{mL}, 1 \mu \mathrm{g} / \mathrm{mL}, 10 \mu \mathrm{g} / \mathrm{mL}, 20 \mu \mathrm{g} / \mathrm{mL}, 50 \mu \mathrm{g} / \mathrm{mL}$ and $100 \mu \mathrm{g} / \mathrm{mL}$, respectively. (g) Relationship between the average fluorescence intensities in the enhanced protein concentration regions and sample's initial concentrations. The data is obtained from the results shown in (a) (f) using the same measurement method illustrated in Figure 5-2 (d) .48 


\section{List of Tables}

Table 4-1 Medium conductivities of different concentrated PBS buffer and corresponding Clausius-Mossotti (CM) factors of protein particles in the suspending medium. The conductivity of the protein particles $\sigma_{\mathrm{p}}$ was taken as $0.0025 \mathrm{~S} / \mathrm{m}$ as a maximum value [70]. 35

Table 5-1 Average values and standard deviations of fluorescence intensity values measured from 15 ROIs along the concentration band as shown in Figure 5-3 (d) indicated using an enlarged image. The same measurement was also applied for Figure 5-3 (b) and (c). The fluorescence intensity data was measured using Nikon imaging software (NIS-Elements AR 3.2).

Table 5-2 Average distances between the concentration band and the constricted channel with varied AC amplitudes, AC frequencies and DC biases. 45 


\section{Chapter 1 Thesis Overview}

\subsection{Research Background}

In recent years, breakthroughs in micro-fabrication technology have greatly increased the speed of new technological development and sharply reduced costs [1]. A manifestation of this potential is in the emergence of 'Lab-on-a-chip' microfluidic devices, which have been making a significant impact on the traditional bioanalytical systems because of their benefits such as reduced material consumption and sample volume requirement, improved reaction efficiency and the potential for enhanced sensitivity and multiplexed analysis [2]. Microfluidic systems refer to set-ups which are employed for the manipulation of liquids and gases in channels that have cross-sectional dimensions generally on the order of micrometers [3]. They can be employed for the manipulation of micro/nano scaled particles in both stationary and flowing liquid environments for different applications. Since most biological analyses and chemical reactions involve fluid transport, microfluidics is of great significance. More importantly, it is possible to incorporate them with other techniques and components to achieve various system functionalities [4]. As a consequence, microfluidic systems have been increasingly used in a wide range of applications, including biological analysis, biochemical detection, chemical synthesis, biomedicine, and drug delivery [5].

However, microfluidics based analytical techniques still face some obstacles. In particular, the extremely small volumes generally required for chip-based analyses reduce the quantity of molecules present in the sample, while these low-abundance biomolecules (proteins, peptides, or micro RNAs) have been generally recognized to serve as indicators for normal biological processes, pathogenic processes, pharmacologic responses to therapeutic intervention, and the 
risk or the presence of a disease, which need to be identified and detected with high sensitivity for further diagnostic purposes [6]. This constraint can be further deteriorated by the practical demand for processing these biomarkers which normally exist in complex biologically relevant mixtures at concentrations beyond the lower limits of detection technologies, such as their concentrations are usually at much lower levels than the threshold of sensors [7, 8]. To address this problem, it is necessary to incorporate sample preparation steps. Sample enrichment is one of the most common sample preparation strategies due to the need to amplify low-concentration biomarkers, especially for miniaturized biosensing and analyzing systems [9]. Therefore, crucial techniques which require low sample volumes with the ability to concentrate biomolecules are demanded.

As a non-destructive particle manipulation method, dielectrophoresis (DEP) has been applied in microfluidic-based devices to manipulate and concentrate bioparticles, including cells [10-12], bacteria [13, 14], viruses [15], DNA [16, 17], and proteins [18, 19]. Specifically, DEP occurs when the particles respond to an inhomogeneous electric field, where the particles in aqueous solution become polarizable thus exhibiting an induced dipole moment. Since the DEP response for a bioparticle is based on its intrinsic properties, it can serve as a label-free analytical method which is important when further processing and characterization steps are necessary. Such a versatile applicability makes DEP an attractive tool to facilitate the analysis of biological particles when used in combination with other conventional analysis techniques and platforms $[20]$.

\subsection{Research Objective}

The main objective of this research is to develop an insulating constriction DEP based microfluidic device which can achieve rapid protein biomolecular concentration effectively 
under regular experimental conditions, with the ultimate goal to integrate the devised DEP concentration module into a portable biosensing system applied for biomarker detection and measurement that could facilitate the practical point-of-care applications and the assessment of one's health status, disease onset and progression in the future [21]. Specifically, the research focuses on:

1) In depth study of DEP mechanism and other related electrokinetic phenomena existing in the microfluidic surrounding with the purpose to obtain an ideal operational strategy for the desired protein concentration effect. It is necessary for the proposed molecular concentration strategy to meet some basic requirements when applied to the device, including a good demonstration of the results for observation and further analysis, plus the capability to obviate the inherent adverse effects or drawbacks within the device during the concentration process.

2) Developing the structure of the insulating DEP based device and formulate the device fabrication procedures accordingly. The designed microstructure of the device need to help achieve effective protein concentration with the proposed strategy under regular, non-extreme laboratory experimental conditions. Meanwhile, the developed microfluidic device should be fabricated with simpler techniques and replicated conveniently for cost reasons. In addition, the availability in module integration for further biomarker sensing and detection processes is also another major consideration.

3) Exploiting the hiding effects of the electric field parameters applied on the device and the medium conductivity of the protein samples on the concentration responses by setting up a series of experiments. Through the analysis and comparison of these experimental results, it is possible to obtain an optimal condition for implementing protein concentration, which makes the 
proposed microfluidic device a more practical option for sample preparation for the potential biomedical applications.

\subsection{Significance and Novelty}

Ideally, a microfluidic system should be self-contained, simple to use, flexible, robust and its fabrication should be uncomplicated [22]. The total cost of a microfluidic system used for bioassays is mainly determined by the complexity of its configuration, the materials and techniques applied in fabrication, the equipment and strategies employed during the sensing and testing period. Considering these influencing factors, in this thesis work we managed to develop an iDEP-based microfluidic device that is capable for protein concentration with a reduced overall cost through introducing novel designing ideas in the following aspects:

\section{1) Fabrication of insulating constriction}

Typically, in terms of the DEP based microfluidic devices related to our work, insulating constriction structures are commonly adopted because a high electric field gradient can be generated over the entire cross section of the constriction [23, 24], since such a feature is critical

for an efficient molecular concentration and quantitative analysis of the concentrated particles [25]. The materials selected for the device fabrication are mostly glass or organic polymers with easy acquisition, e.g. polydimethylsiloxane (PDMS), especially for the post array constrictions $[16,26]$ and triangular constricted structures [17]. However, the only obstacle for reducing the total cost of such device is that fabricating submicron/nano-scaled constriction usually requires high-resolution lithographic techniques, such as electron beam lithography (EBL) or focused ion beam lithography (FIB). Both techniques are very time consuming, low throughput and high-cost $[27,28]$, and are quite challenging to create the constriction together with other parts of the 
device like sample loading, fluid transport channel and detection chamber in simple fabrication steps. Besides, nanopipettes fabricated by laser pipette puller technique have been reported earlier for the biomolecules DEP trapping [29]. Although the nanometer sized concentration device can be fabricated without using conventional nanofabrication method and specialized fabrication facilities, it also has inevitable limitations for multiplexing biosensing and integration with a microfluidic device for molecule concentration. Under the circumstances discussed above, an alternative technique for fabricating the submicron/nano-scaled constriction is very desirable in order to address the necessity of reducing the fabrication cost of the microfluidic device and meanwhile to provide the possibility of simple integration with other devices involving different functions.

The research work in this thesis demonstrated a microfluidic device fabricated by the techniques of DNA molecular combing and soft lithography, which could create a sub-micron sized constricted channel embedded within a microchannel. This idea was directly inspired by the recently reported application of nanochannel electroporation (NEP) for precise drug delivery, in which the DNA combing and imprinting (DCI) method was introduced to form a sealed array of laterally ordered nanochannels interconnected to microchannels with controllable sizes and rounded shape $[30,31]$. The fabrication process and integrated NEP device is illustrated in Figure 1-1. This technique was originally applied for producing large laterally ordered nanochannel and microwell arrays over an area of several square centimeters to form a nanoscale transport system [32]. 


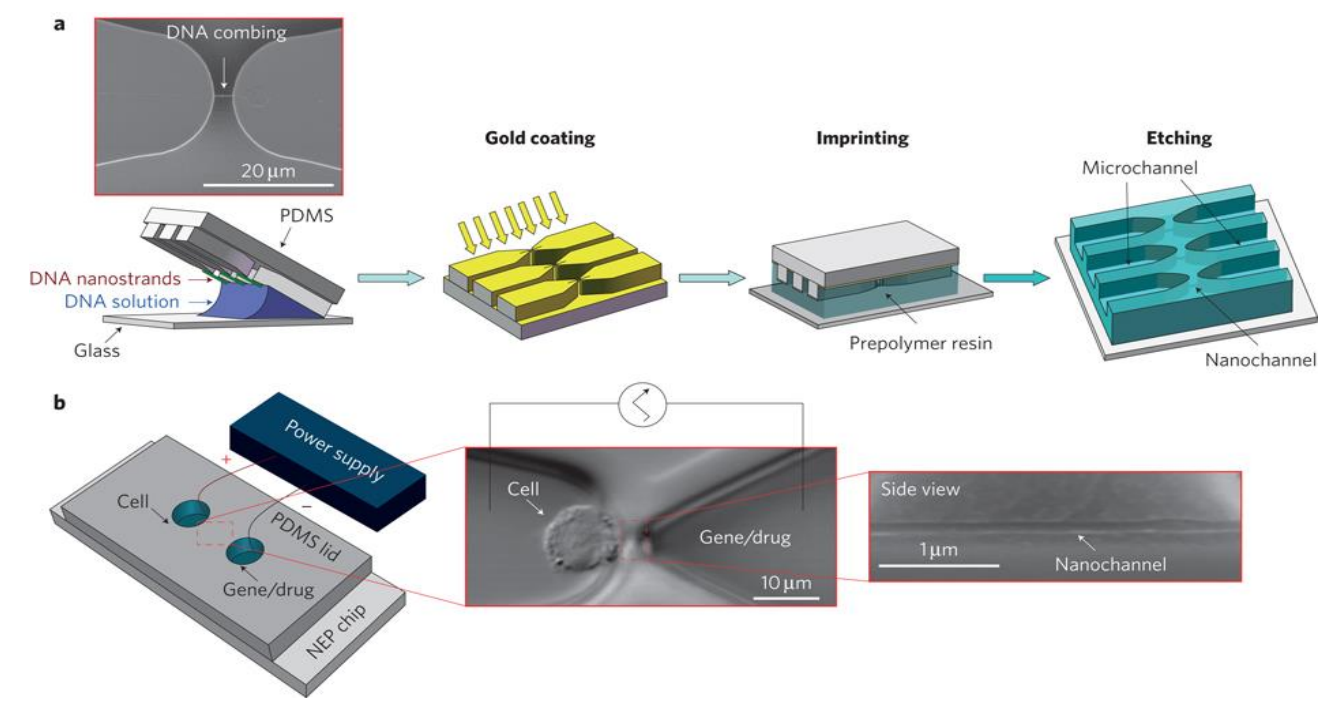

Figure 1-1. Fabrication and operation of the NEP device. (a) Schematic showing the fabrication of the NEP chip by DCI. (b) Left: schematic of an NEP chip covered by a PDMS lid with electrodes placed in reservoirs. Middle: optical micrograph of a cell in the left microchannel and positioned at the tip of the nanochannel using optical tweezers. The right microchannel contains gene or drugs. Right: SEM image of side view cut of a nanochannel. The nanochannel is $\sim 90 \mathrm{~nm}$ in diameter and $\sim 3 \mathrm{~mm}$ long [30].

Molecular combing is a technique for stretching, aligning, and immobilizing coiled DNA molecules in a solution onto a flat surface through a dewetting process [33].The stretched DNA molecules could be transferred onto other solid surfaces by contact printing, allowing for the generation of more complex patterns. The most notable advantage of this fabrication method is that it eliminates the need for e-beam lithography or other expensive and time-consuming techniques to fabricate the sub-micron scale channel. Throughout the fabrication procedures, only the stamp molds fabrication and gold coating need to be conducted in a cleanroom, the other steps could be easily handled in a regular lab. Furthermore, once the device mold is ready, it can be reused for a long period of time to generate at least hundreds PDMS stamps for molecular combing by common soft lithography methods, which significantly reduces the overall cost for device fabrication. According to the recent experimental results, molecular combing is 
well proved to be an effective, low-cost and high throughput fabrication technique without sacrificing the accuracy of the designed device structure.

2) Method and realization of protein concentration

Practically, a highly focused electric field and a strong field gradient could be generated in the area adjacent to the insulating constriction embedded in a sealed channel when an AC or DC electric field is applied across the constriction. Under such condition, positive dielectrophoresis (pDEP) or (b) negative dielectrophoresis (nDEP) is obtained and further applied for trapping particles. Rather than applying solely the AC or DC field to get the DEP trapping effect, in this thesis we proposed a methodology of employing a DC bias coupled AC electric field to acquire an adjustable focusing band for the concentrated protein molecules through a balance of DEP and DC electrokinetic forces. Since the presence of the DC bias influences the force balance condition which occurs at the local potential minimum, by changing the strength of DC bias, the size and location of the formed concentration band can be easily manipulated. This feature provides significant flexibility and reliability for the microfluidic device and would largely facilitate its further application when functioning together with other units in an integrated labon-a-chip bioassay system.

In addition, the electric field was supplied from an external signal source that is connected to the electrodes fabricated on the device. This configuration allows the insulator-based device to work under a relatively low input $\mathrm{AC}$ voltage, which eliminates the need for the accessories such as voltage amplifier so that a general function generator would be enough for tuning the input electric field. Therefore the developed device offers higher feasibility since its functions can be realized under a simpler experimental setup. Meanwhile, in this thesis study low frequency 
(below $10 \mathrm{kHz}$ ) AC signal was applied to achieve protein concentration. Such design provides another potential advantage for the developed device in the bioassays, which is that the required signal for the protein concentration effect could be generated from some unspecialized or customized equipment using their original interface connection, for example the standard headphone jack of a cellphone or tablet which can output an AC waveform up to $20 \mathrm{kHz}$. This property would dramatically enhance the accessibility and universality of developed device for the future application in a portable, low-cost lab-on-a-chip bioassay system.

\subsection{The Outline of the Thesis}

This thesis consists of six chapters. It is initiated with the thesis overview in Chapter One, in which the research background and objectives, as well as the significance and novelty of this research were stated. Following chapter one, five chapters demonstrated the details of the presented research work. Chapter Two is a literature review in the DEP microfluidics devices, including its brief history and the state-of-art of insulator DEP devices for biomolecules applications. Chapter Three described the theory of DEP and the principle of protein concentration applied for the device presented. Chapter Four introduced the design of the insulating constriction DEP based microfluidic device and demonstrated fabrication procedures and testing methodology in detail. Chapter Five characterized the protein biomolecules concentration effects with the combined analysis of the electric field parameters including DC bias, AC amplitude and frequency, as well as the influence of medium conductivity. The measurement sensitivity of the concentrated protein using the developed device was also discussed. Conclusions and future directions are summarized in Chapter Six. 


\section{Chapter 2 Literature Review}

\subsection{Dielectrophoresis-based Microfluidic Device}

The serious scientific study of dielectrophoresis did not begin until the 1950's. Herbert A.Pohl was the first to truly study dielectrophoresis, and provided the principal governing force equation still accepted today [34]. This equation is discussed further in Chapter 3 where it appears as Equation (3). The majority of these past studies of dielectrophoresis either attempt to explain elements of the underlying physics or apply the technique of dielectrophoresis to accomplish a particular goal such as cell manipulation or the determination of a particle's electrical properties. The application studies seldom contain substantial theoretical examinations of their setups. A fairly comprehensive overview of the development of this field up to the present is compiled by Ronald Pethig in 1996 [35]. He wrote a review article taking many of the published works available and summarizes most of the relevant factors influencing the dielectrophoretic behavior of particles in solution that have been elucidated by these works. The characteristics of dielectrophoresis make it appealing for analytical applications such as fractionation, pre-concentration, and separation methods [20]. From then on, with the urgent need for molecular biology related microanalysis with much higher sensitivity and greater throughput, as well as the fast development of micro-electro-mechanical systems (MEMS), scientists have been putting increasing efforts in the study of exploiting the advantages of dielectrophoresis in biomolecular analysis and the combination of dielectrophoresis with microfluidic systems for a variety of biomedical and biotechnological applications such as the precise monitoring and manipulation of various biomarkers.

The potential of DEP for protein manipulation and concentration has been recognized and pioneered by Washizu et al. more than 20 years ago [36]. In their work, the possibility of 
fractionation of proteins was outlined as well as some fundamental characteristics of biomolecular DEP. Follow up work mainly by Hughes, Morgan, and coworkers showed a more detailed investigation of selected proteins on patterned microelectrodes as well as a characteristic frequency dependence of protein DEP [37, 38]. In the last decade, the interest in protein DEP intensified due to the demand for protein analysis tools as well as the improvement of micro- and nanofabrication techniques applied to protein DEP [39].

Briefly, in order to generate a dielectrophoretic force within a microfluidic device, a nonuniform electric field is required. Typical strategies include either patterning particular size/shape of the electrodes, or changing the microchannel's opening size dramatically, or placing insulator structures (and/or arrays) in the microchannel [20]. Consequently, many different configurations were employed for the design of DEP-based microfluidic systems. Generally two methods have been used to generate the necessary non-uniform electric fields for producing a DEP force: electrode-based DEP devices and insulator-based devices [40]. Examples representing the two methods are shown schematically in Figure 2-1 [41].
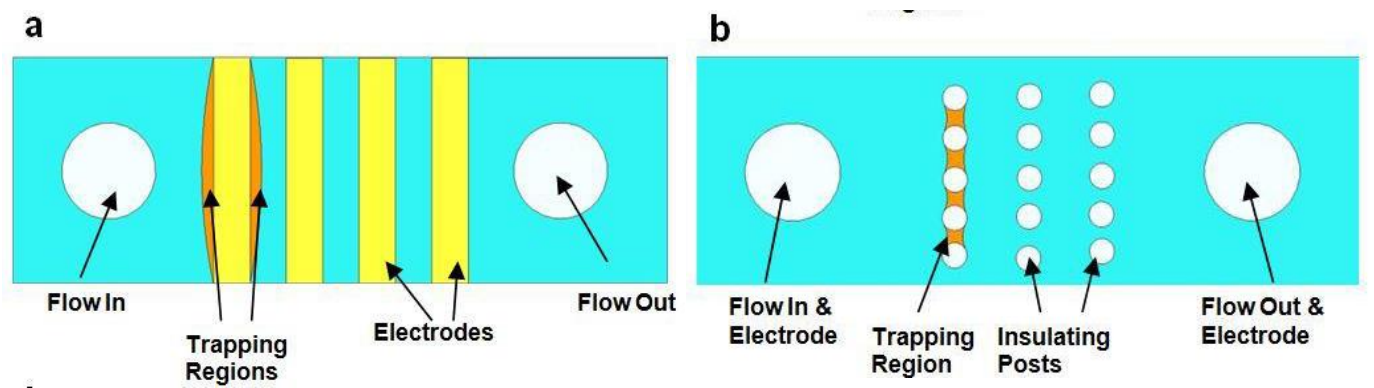

Figure 2-1. Examples of eDEP and iDEP structures. (a) eDEP design consisting of rectangular electrodes with trapping regions shown. (b) iDEP design consisting of circular insulating posts with trapping regions shown. 
DEP devices using electrodes to generate inhomogeneous electric field are often called electrode-based DEP (eDEP). According to the previous work reported, eDEP devices are mostly fabricated by photolithography [42], electron-beam evaporation [43], chemical vapor deposition (CVD) [44], etc. The shapes of the electrodes include the following: single pair electrodes [45], quadrupole electrodes [42], nano-tip electrodes [46], castellated electrodes [47] and arrays of micro-electrodes [48], etc. Figure 2-2 shows the structures of micro tip and castellated electrode arrays [49].

Although these device configurations allow for micro/nanoelectrodes generating high electric fields with low applied voltage, the fabrication is often complicated and expensive, making large-scale systems less economically feasible [16]. Furthermore, electrolysis can occur due to the high voltage applied, and the electrode functionality is prone to decrease with fouling when working with biological samples, rendering the devices to be hardly reusable.
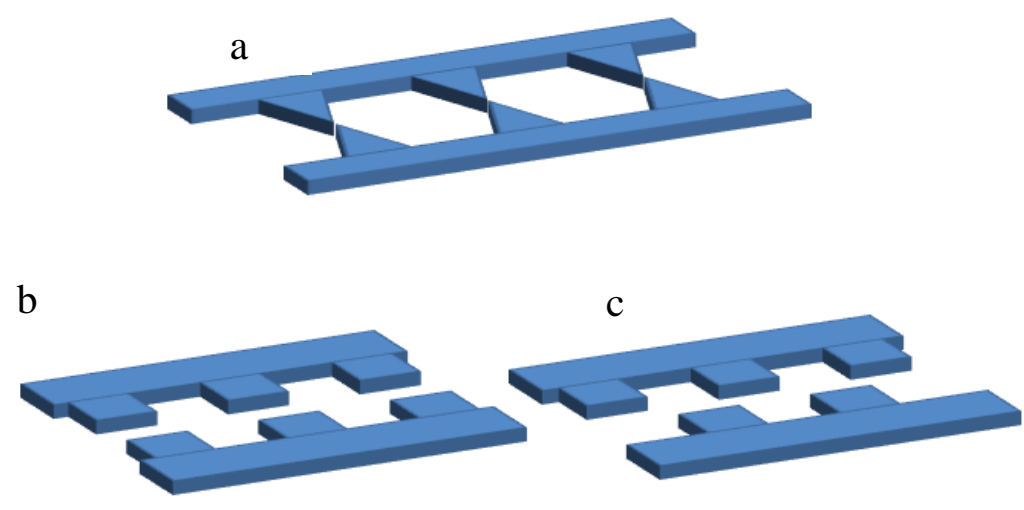

Figure 2-2. Shapes of eDEP device: (a) Micro tip electrode array. (b) and (c) Castellated electrode arrays with normal and shifted configurations.

Insulator-based DEP (iDEP), also called electrodeless DEP, is gradually employed as an alternative to the classic electrode-based DEP. In an iDEP device, external electrodes are employed to generate a uniform electric field, and insulating microstructures are embedded in the microchannel to squeeze the electric field. Thereby, a high electric field gradient with a local 
maximum is created. This technique allows a significantly enhanced local electric field gradient generated at the constriction within a device made of an insulating material and running in a conductive medium [25]. Compared to electrode-based DEP, the electrodes are placed in distal inlet and outlet reservoirs and the electric field is defined by channel insulators and the conductive media. This resolves many of the issues encountered with eDEP (electrolysis, bubbles, fouling). Therefore the iDEP devices are more mechanically robust and chemically inert because insulators are able to maintain their functionality despite surface fouling [50]. Furthermore, iDEP's fabrication process is usually much less complicated [51]. For example, photolithographic methods [52] or etching methods [17] were applied in the process. To improve the gradient of electric field, methods such as focused ion beam milling (FIBM) have also been used to improve the efficiency to manipulate samples [6, 53].

\subsection{State-of-Art Development of iDEP devices}

iDEP was theoretically conceived and proof of concept established in the early 2000s [54]. Since then many different types of insulators have been utilized to achieve particle sorting or trapping, including single insulating obstacles [55], multiple insulating posts in arrays [56, 57], and modified channel geometry, as shown in Figure 2-3 [58]. Examples of insulator shapes currently utilized include rectangle [59-61], triangle [62, 63], sawtooth [64-66], circular posts $[16,54,57]$ and diamond posts $[67,68]$. For nearly all the iDEP designs, the constriction geometry defines the overall performance, whether the basic shape is repeated or varies in some characteristic dimension. Work has also been performed by forming a ridge inside microchannel as a height constriction to obtain 3D iDEP [7, 69], which also highly increased the electric field near the insulating structure. 
With the help of iDEP microfluidic devices, molecular sorting, trapping or separation could be effectively realized, which significantly improves the efficiency of sample preparation and provides the potential for complicated biological assays. Considering the proposed objective of this research, which is to achieve protein molecular concentration in an iDEP microfluidic device as a sample preparation process for the potential biosensing application, in this section three typical state-of-art iDEP device configurations reported in the previous literature which were mainly applied for biomolecular trapping and preconcentration will be reviewed and discussed.

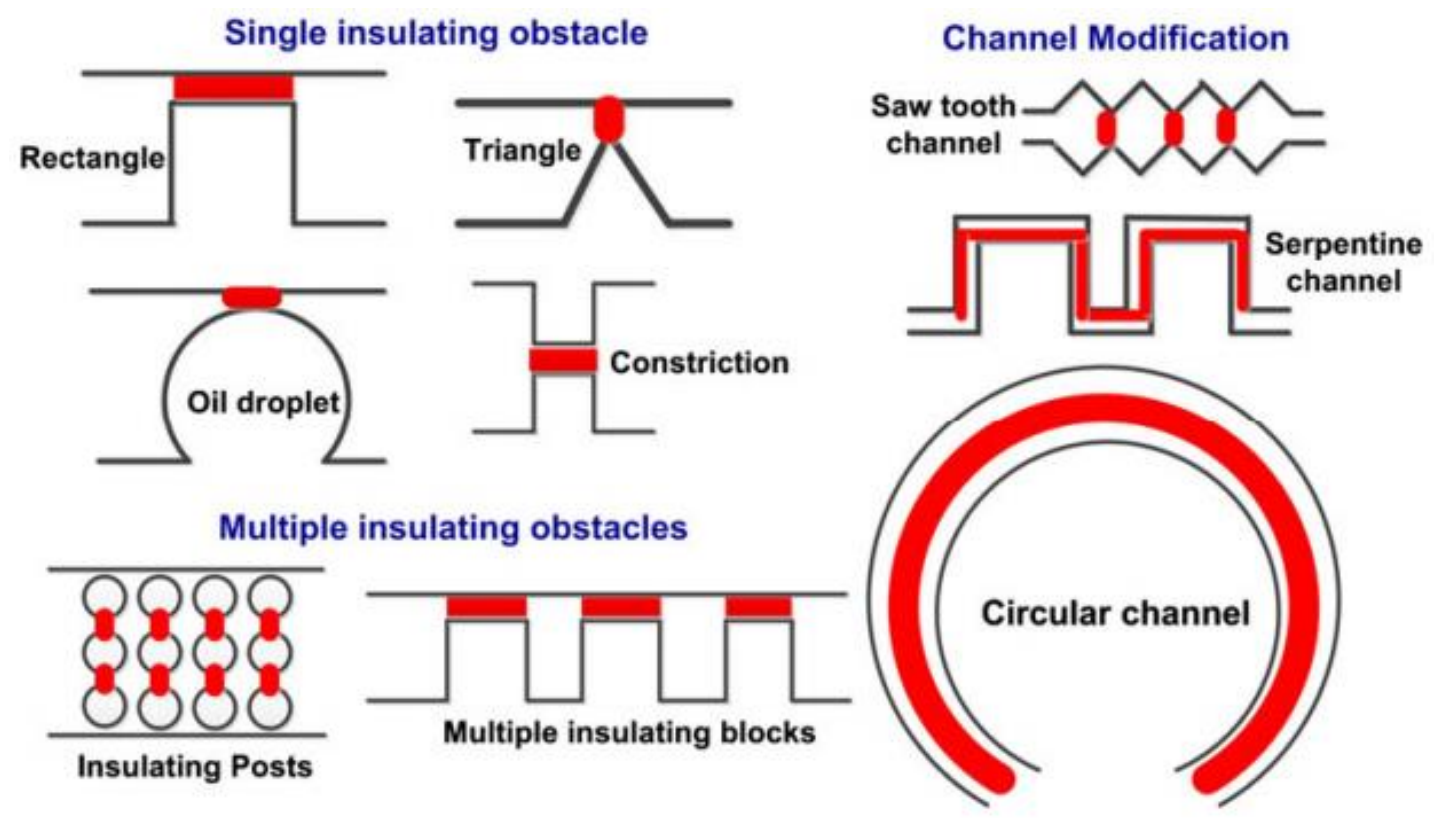

Figure 2-3. Common insulating obstacle geometries reported in the literature. Red zones indicate where the particles experience the strongest dielectrophoretic effect.

\subsection{1 iDEP devices with insulator post arrays}

Placing insulator post arrays in the microfluidic channel of an iDEP device was attempted by many research groups. Initial work on trapping single- and double-stranded DNA with DCiDEP was conducted by Chou et al. in 2002 [17]. They used blocks to squeeze electric field iDEP to trap and concentrate single and double-stranded DNA, as shown in Figure 2-4. DNA trapping 
was demonstrated using insulating constrictions at relatively low frequencies. Later LapizcoEncinas et al. reported protein trapping using an iDEP device, as shown in Figure 2-5 [70]. The authors showed that BSA could be manipulated via negative DEP under DC condition within a glass microfluidic device containing insulating circular post arrays. The effect of the electric field on the dielectrophoretic response of the protein and the effect of the properties of the suspending medium on the trapping of BSA particles were systematically studied. Strong negative dielectrophoretic trapping occurs at high electric field strengths; by increasing the conductivity and lowering the $\mathrm{pH}$ of the medium, dielectrophoretic trapping can be achieved with an applied voltage of $700 \sim 1600 \mathrm{~V} / \mathrm{cm}$. The maximum protein concentration in the trapping region was observed using the highest conductivity buffer.

(A)

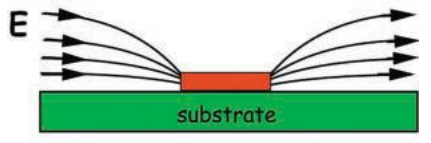

(B)

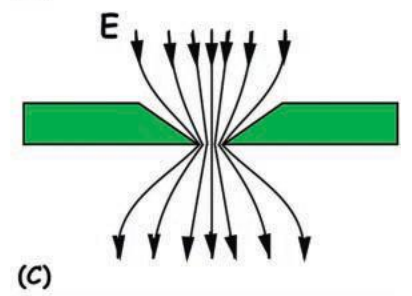

(C)

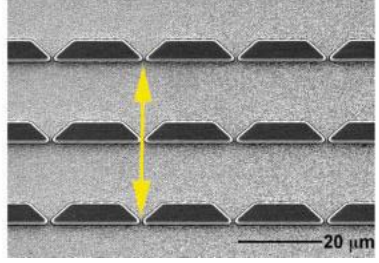

Figure 2-4. Schematic of a microfluidic DEP trapping. (a) A metallic DEP trap made of microfabricated wire(s) on a substrate. The wire(s) may be either free-floating or connected to a voltage source. (b) An electrodeless DEP trap made of dielectric constrictions. The solid lines are electric field lines E. (c) A scanning electron micrograph of an electrodeless DEP device consisted of a constriction array etched in quartz. The constrictions are $1 \mu \mathrm{m}$ wide and $1.25 \mu \mathrm{m}$ deep. The whole chip measures $1 \times 1 \mathrm{~cm}$. The applied electric field direction $\mathrm{z}$ is shown by the double-headed arrow. 


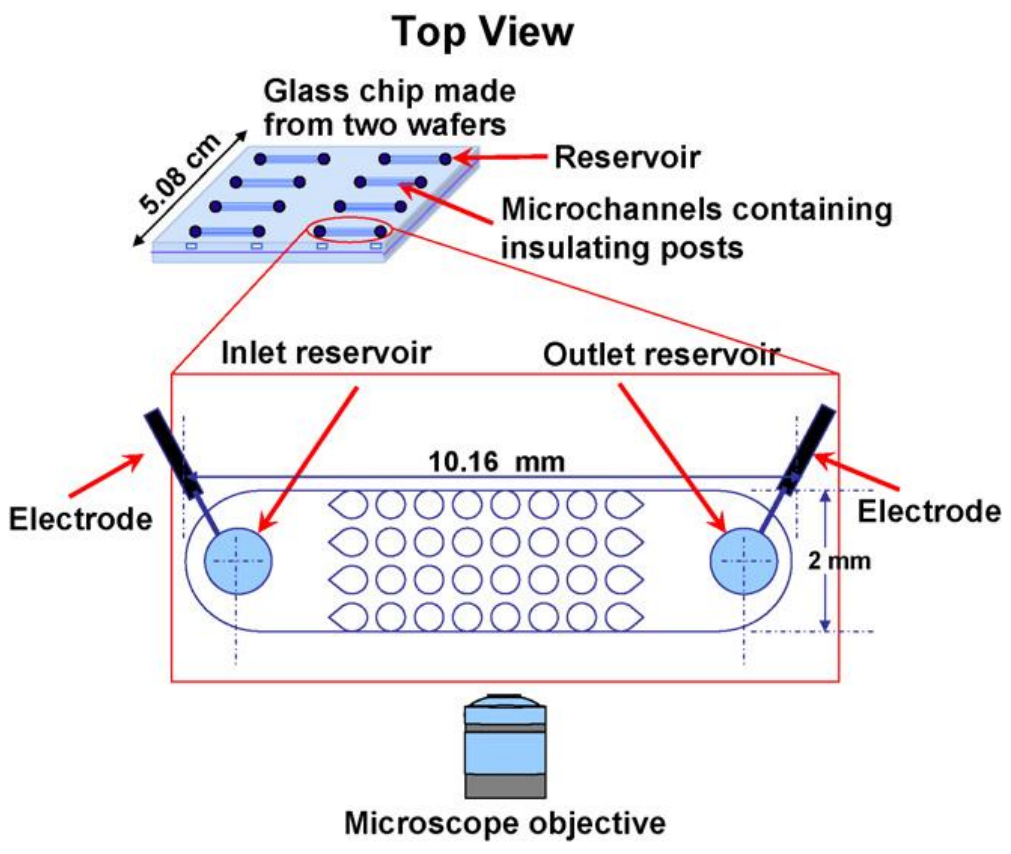

Figure 2-5. Schematic of the microfluidic device and a magnification of the microchannel containing insulating circular post arrays.

Furthermore, Staton et al. fabricated insulating constrictions in a single microfluidic channel with varying sawtooth shape to construct an iDEP system, as shown in Figure 2-6 [71].With a DC-iDEP device they showed rapid and selective concentration of the amyloid-beta (A $\beta$ ) species. While $A \beta$ monomers exhibited streaming DEP, $A \beta$ fibrils were captured and concentrated at the narrow constriction of the sawtooth structure demonstrating iDEP trapping.

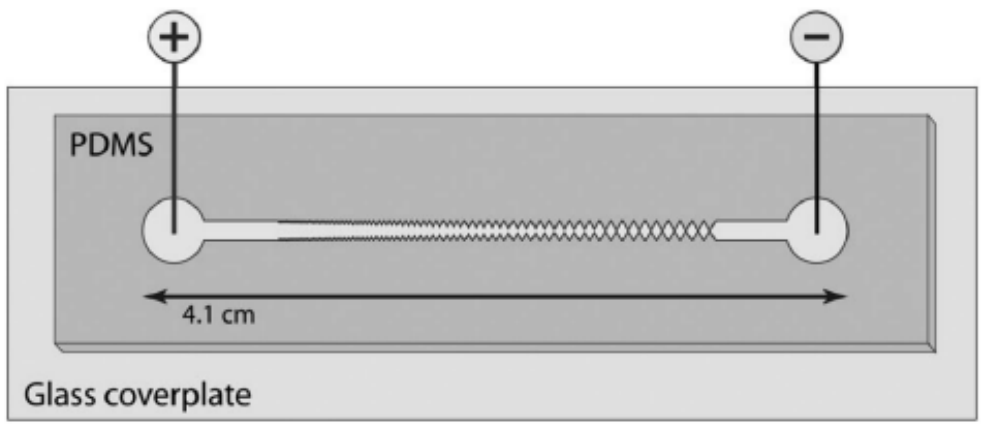

Figure 2-6. Schematic of the direct current insulator gradient dielectrophoresis device. An insulated sawtooth pattern is integrated with a tapered microfluidic channel to generate localized non-uniform electric field gradients of increasing strength from left to right. 
However, one typical drawback is it usually requires large voltage potentials (typically around $1000 \mathrm{~V}$ ), and a high electric field in conductive biological fluids inside a microchannel [57, 72], which always causes high electric current leading to a large heat buildup and Joule heating effects near the insulating structures in direct current power supply devices [51].

\subsection{2 iDEP devices with nanopipettes}

Another iDEP approach can be achieved by employing a nanopipette used by Clarke et al. to create narrow openings and demonstrate the trapping of two proteins, protein $G$ and immunoglobulin $\mathrm{G}(\mathrm{IgG})$ [73]. The tip of this nanopipette has a $100 \sim 150 \mathrm{~nm}$ internal diameter creating an electric field strength of $10^{6} \mathrm{~V} / \mathrm{m}$. Using a physiological buffer to retain protein integrity, a maximum of 3000-fold protein concentration was achieved due to positive DEP with reversible protein accumulation, as depicted in Figure 2-7. Moreover, the same device was used to measure protein conductivity [74]. However, this approach could introduce certain limitations in the potential purpose of integration in a complex microfluidic system, due to the 3-D based geometry of the nanopipette configuration which may reduce its compatibility with other units compared with other planar iDEP structures.

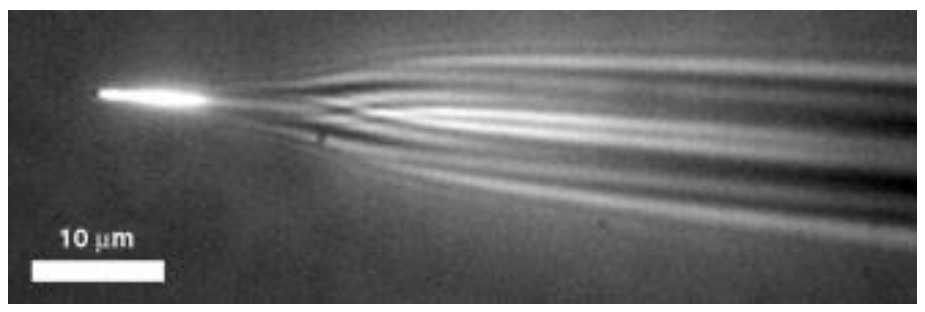

Figure 2-7. Fluorescence image recorded by using wide-field illumination $(5.2 \mathrm{~mW})$ of a nanopipette filled with $100 \mathrm{nM}$ Alexa-488 dye in PBS buffer 


\subsection{3 iDEP devices with triangular constrictions}

In 2003, Chou et al. published an article on the construction of DEP device for a lab-ona-chip micro total analysis system ( $\mu \mathrm{TAS}$ ), which brought about a novel concept of using triangular dielectric constrictions to achieve molecular DEP trapping with AC electric field [75]. In this article, the differences between electrodeless trapping via insulating constrictions and conventional trapping via metal electrodes were discussed. Several examples of the application using this device, including DNA trapping and cell sorting were also demonstrated, as shown in Figure 2-8.

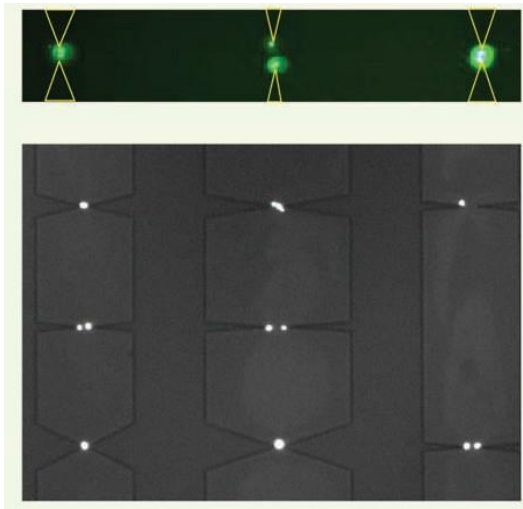

(a)

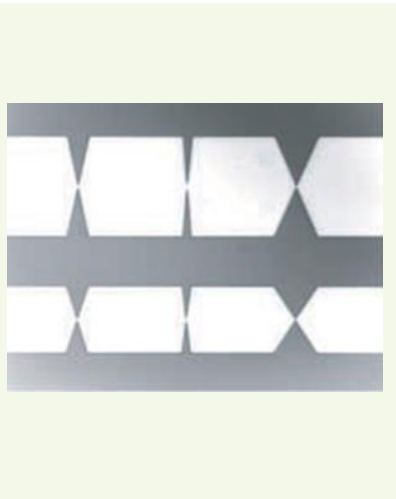

(b)

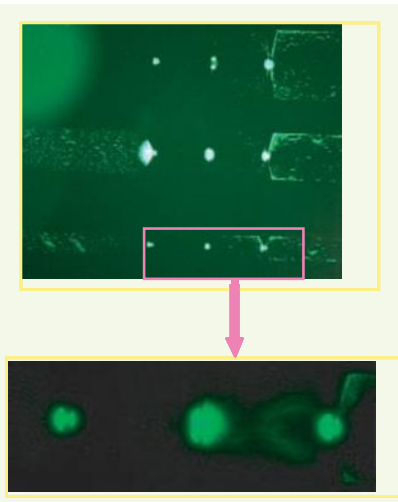

Figure 2-8. (a) Fluorescein-labeled single-stranded $1 \mathrm{~kb}$ DNA and double-stranded T5 DNA (103 $\mathrm{kbp}$ ) trapped at a PDMS constriction array in 1x PCR buffer.. The constrictions are depicted by their triangular contours. The applied field was $200 \mathrm{Vpp} / \mathrm{cm}$ at $1 \mathrm{kHz}$ in $0.5 \mathrm{x} \mathrm{TBE}$ buffer. (b) iDEP device for cell capture and enrichment. The constriction traps was fabricated in silicone elastomer using soft lithography sealed against a glass slide and have a $4 \mu \mathrm{m}$ opening and are 10 $\mu \mathrm{m}$ deep, with channel width ranging from 100-250 $\mu \mathrm{m}$ and various radius of curvature. The images on the right show the trapping of GFP-expressing E. Coli in a buffer with $50 \mathrm{mM}$ histidine, $250 \mathrm{mM}$ sucrose. The applied field was $300 \mathrm{Vpp} / \mathrm{cm}$ at $1 \mathrm{kHz}$.

Most recently Liao and Chou reported iDEP devices with nanometer-scale constrictions fabricated by a combination of photolithography and electron beam lithography using the DEP trapping strategy called 'molecular traps and dams', as depicted in Figure 2-9 [76] and further employed in the following work [77]. The numerical simulations suggested that with a $100 \mathrm{~nm}$ 
constriction device the DEP force acting on a streptavidin molecule reaches $10 \sim 100 \mathrm{pN}$, whereas less than $10 \mathrm{pN}$ DEP force is generated with $1 \mu \mathrm{m}$ constrictions. In this work, they used a physiological buffer to maintain the protein's conformation and functionality, which poses a challenge due to significant Joule heating. However, using a $30 \mathrm{~nm}$ nano-constriction device at 200 300 Vpp/cm AC voltage and $1 \mathrm{MHz}$ frequency combined with a slight DC offset, concentration of the protein streptavidin was obtained due to negative DEP at regions of low electric field strength. The authors specifically selected a frequency range for negative DEP so that the protein would be trapped at the low electric field regions where a rise in temperature is less significant. With this well-designed device, they achieved a $10^{5}$-fold concentration increase within 20 seconds, as shown in Figure 2-10 [76].

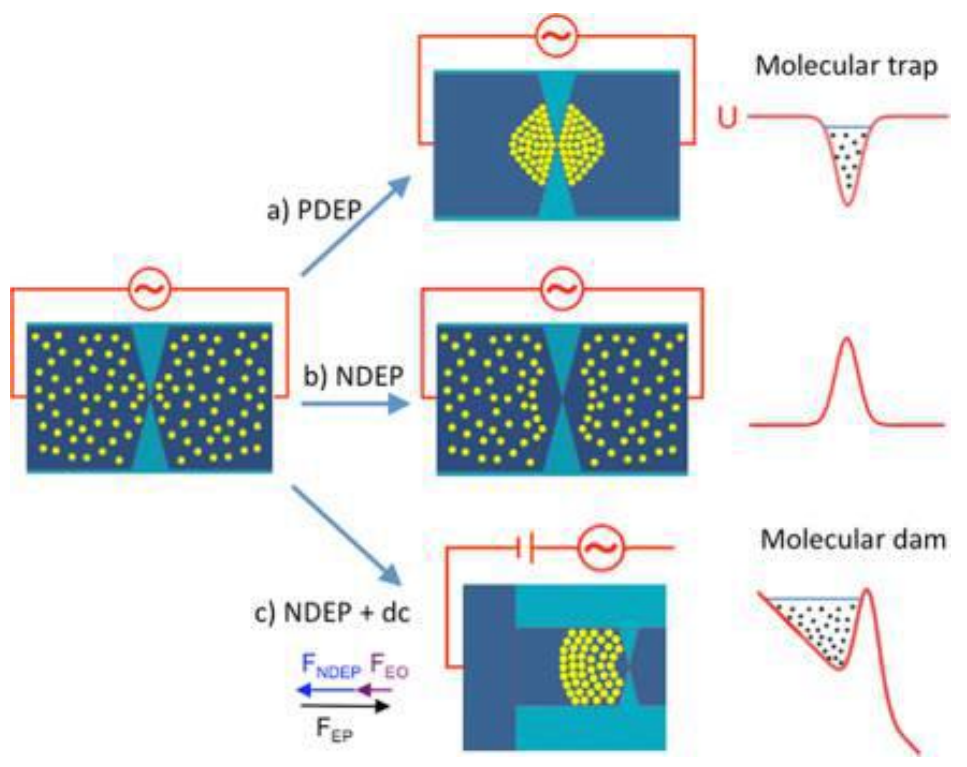

Figure 2-9. Concept of nanoscale molecular trap and dam. (a) Positive dielectrophoresis (pDEP). (b) Negative dielectrophoresis (nDEP). (c) Molecular trap and dam via the combination of nDEP and a DC bias. 


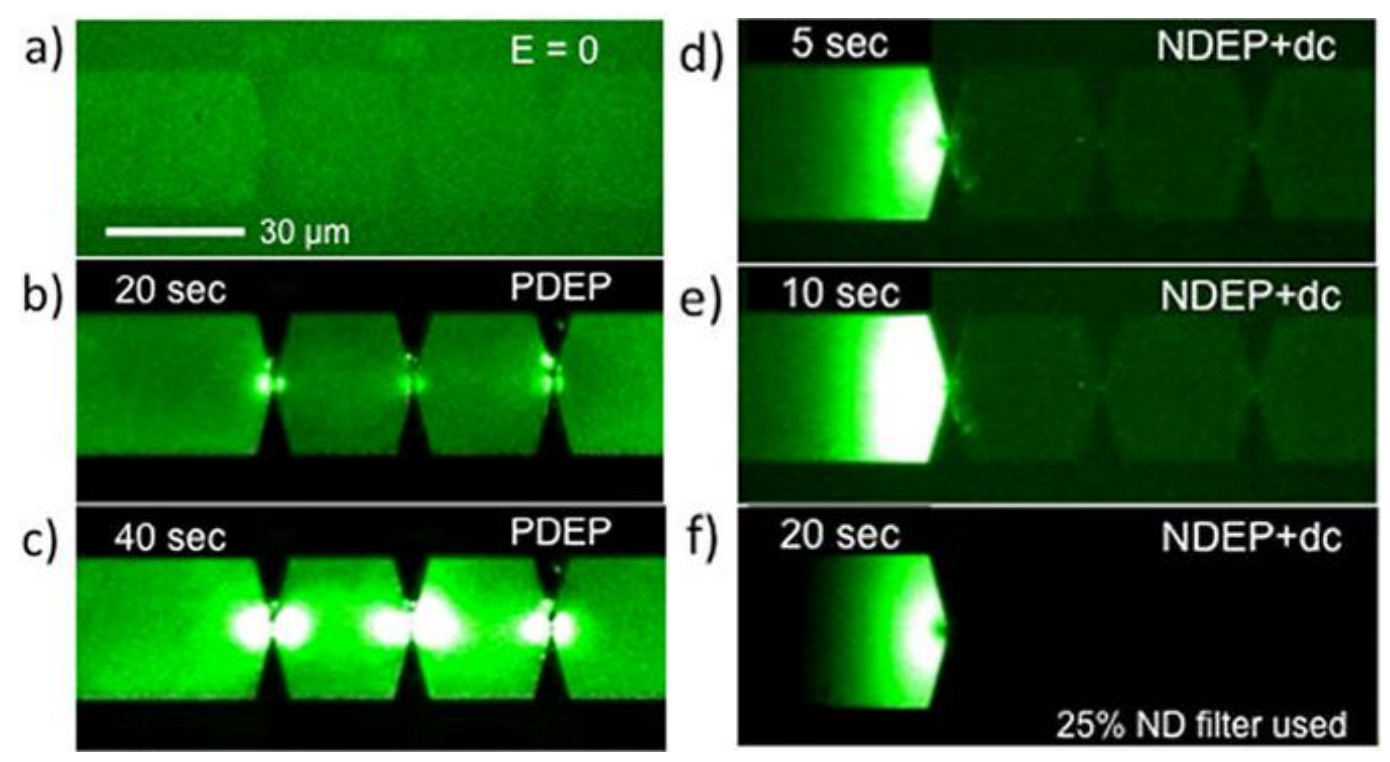

Figure 2-10. Protein trapping and damming with nanoscale eDEP. (a) Proteins (Alexa-488 streptavidins, $10 \mu \mathrm{g} / \mathrm{mL}$ ) loaded in the chip by capillary force trapping with pDEP. (b) 20 and (c) $40 \mathrm{~s}$ after $473 \mathrm{Vpp} / \mathrm{cm}$ ac field applied across the chip at $10 \mathrm{kHz}$. This field is focused $7 \times 10^{4}$-fold $\left(\sim 3.3 \times 10^{9} \mathrm{Vpp} / \mathrm{m}\right)$ over the applied field at the constrictions. All three constrictions show trapping of streptavidins, indicating PDEP is at work. (d) (f) Protein damming demonstrated by $\mathrm{nDEP}+\mathrm{DC}$ : ac field of $214 \mathrm{Vpp} / \mathrm{cm}$ is applied at $1 \mathrm{MHz}$ with $1.5 \mathrm{~V} / \mathrm{cm}$ DC bias (with positive potential at the right side of the channel). The dark zone at the center of the first constriction indicates NDEP is at work where streptavidins are repelled.

Compared to the other 2 types of insulator structures discussed above, iDEP devices with triangular constrictions scaling down to nanometers possesses huge advantages when used for biomolecular trapping and enrichment, since they achieve high level concentration from the raw sample without the demand to build up high DC electric field, resulting in a much simpler experimental set-up. As a sample preparation device, it also provides effective access to the integration with other microfluidic modules for further sample analysis. However, the only limitation of this strategy lies in that the fabrication of nanometer-sized constrictions still requires the techniques like e-beam lithography with higher complexity in order to guarantee the accuracy and high sensitivity of the designed iDEP device, which still requires overcoming the obstacles of high cost and the requirement for expertise knowledge. 


\subsection{Chapter Summary}

In summary, a number of iDEP microfluidic devices with various configurations have been developed for biomolecular trapping and preconcentration, using materials ranging from glass to polymers. Each of these device has demonstrated its advantageous characteristic, yet they are still not capable to meet the multiple demands of biomedical point-of-care applications in practical use, such as high efficiency, applicability to microfluidic system integration and low cost simultaneously. The status quo of the development in DEP-based microfluidic devices inspired us to propose a design with improved strategy that could combine all the features that meet the requirements mentioned above in a single microfluidic device. Hopefully it would be available to fulfill the functions of sample preparation and device integration under low-resource laboratory settings, which offers greater potential for playing a key role in a micro-total-analysis system ( $\mu$ TAS) for biological analysis. 


\section{Chapter 3 Dielectrophoresis Theory and Principle of Protein Concentration}

\subsection{Dielectrophoresis}

Dielectrophoresis (DEP) refers to the migration of a polarizable particle in an inhomogeneous electric field $(\boldsymbol{E})$. When a dielectric particle is suspended in an electric field, polarized charges are induced on its surface, which establishes electric dipoles. In a uniform electric field, Coulomb force are generated on both sides of the particle are equal in magnitude and opposite in direction, resulting in zero net force on the particle. However, when the electric field is non-uniform, the Coulomb forces on either side of the particle can be different, and the overall force results in particle motion. Since the direction of the force is determined by the spatial variation of the field, the particle always moves toward or against the direction of the electric field maxima [4]. The phenomenon can be observed under the influence of either AC or DC electric fields.

For most DEP applications, it is useful to describe the force acting on a polarizable particle generated by the interaction between the induced dipole and the electric field. The DEP force acting on a particle is related to the apparent dipole moment $\boldsymbol{m}$ [78]:

$$
\boldsymbol{F}_{D E P}=(\boldsymbol{m} \cdot \nabla) \boldsymbol{E}
$$

For an induced dipole moment, Equation (1) can also be expressed in terms of the particle polarizability $\alpha$ [78]:

$$
\boldsymbol{F}_{D E P}=\frac{1}{2} \alpha V \cdot \nabla \boldsymbol{E}^{2}(\mathbf{2})
$$


where $V$ denotes the particle volume. In an $\mathrm{AC}$ electric field the direction of the induced force does not change and generally the time average is used for the calculation of the DEP force. In classical DEP theory, the time averaged DEP force for a spherical particle in a medium of permittivity $\varepsilon_{m}$ is given as [78]:

$$
\boldsymbol{F}_{D E P}=2 \pi r^{3} \varepsilon_{m} \operatorname{Re}[K(\omega)] \nabla \boldsymbol{E}_{r m s}^{2}(\mathbf{3})
$$

where $r$ is the particle radius, $\omega$ is the angular frequency, $\boldsymbol{E}_{r m s}$ is the root mean square electric field, and $\operatorname{Re}[K(\omega)]$ is the real part of the Clausius-Mossotti factor given as:

$$
\operatorname{Re}[K(\omega)]=\frac{\varepsilon_{p}^{*}-\varepsilon_{m}^{*}}{\varepsilon_{p}^{*}+2 \varepsilon_{m}^{*}}(\mathbf{4})
$$

Here, $\varepsilon_{p}^{*}$ and $\varepsilon_{m}^{*}$ denote the complex permittivities of the particle $(p)$ and medium $(m)$, respectively. The complex permittivity for the particle (and similarly for the medium) is given by $\varepsilon_{p}^{*}=\varepsilon_{p}-j \frac{\sigma_{p}}{\omega}$, where $\sigma_{p}$ denotes the particle conductivity.

The Clausius-Mossotti factor is characteristic for the DEP response. Depending on the sign of the Clausius-Mossotti factor, particles are attracted to the regions of highest electric fields or repulsed from those regions as shown schematically in Figure 3-1 [40]. These two cases refer to positive DEP (pDEP) and negative DEP (nDEP), respectively. Equation (3) demonstrates the frequency dependence of the dielectrophoretic response of a sphere and allows estimating the resulting forces in electric field gradients. At high frequency, $\boldsymbol{F}_{D E P}$ is typically governed by the permittivity of the particle and the suspending medium, conversely at low frequency and under DC conditions the Clausius-Mossotti factor is dominated by the conductivity of the particle and the suspending medium. 
Moreover, the frequency dependent change from positive to negative DEP is characterized by the so-called crossover frequency where the Clausius-Mossotti factor reverses its sign. The polarization effects on particles are often studied by investigating the changes in crossover frequency.

(a)

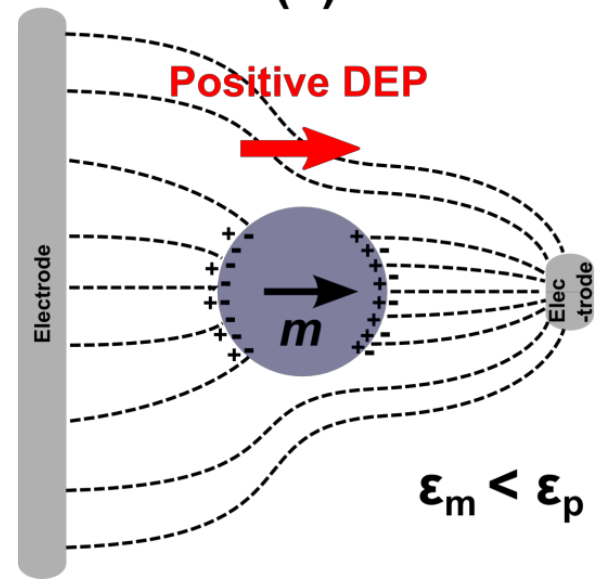

(b)

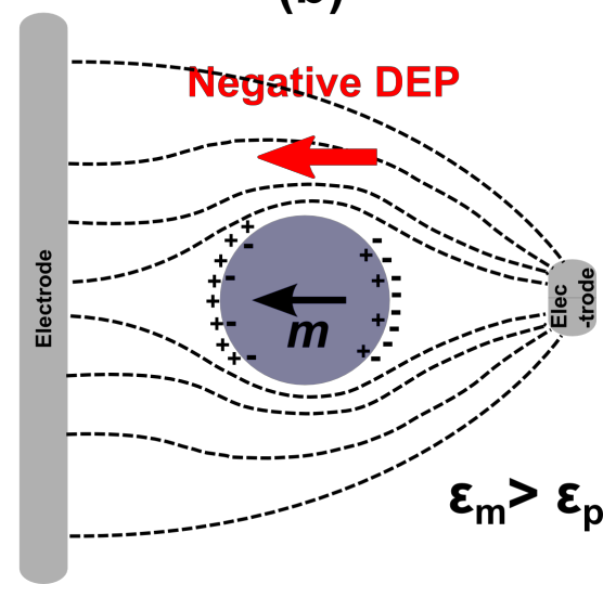

Figure 3-1. Schematic representation of a mechanism of DEP occurring under an inhomogeneous electric field by which a particle is transported (a) toward the higher electric field gradient (positive DEP) or (b) away from it (negative DEP).

Assuming there are two types of particles and media for comparison purposes: particles type A with a conductivity of $\sigma_{p}=1 \mathrm{mS} / \mathrm{m}$ (typical value for polymer particles when suspended in water) and particles type B with a ten-times lower conductivity of $\sigma_{p}=0.1 \mathrm{mS} / \mathrm{m}$; medium type A with a conductivity of $\sigma_{m}=1 \mathrm{mS} / \mathrm{m}$ (close to the conductivity of DI water) and medium type B with a hundred-time lower conductivity of $\sigma_{m}=0.01 \mathrm{mS} / \mathrm{m}$. The relative permittivities of particles were assumed to be 2 (close to a typical polymer) and the permittivity of media was assumed to be 100 (close to DI water), respectively. Two sets of calculations were performed. Case one: for particles type A and B which are suspended in media type B. Case two: for particles type A which are suspended in media type A and B. The values of the $K(\omega)$ (real and 
imaginary parts) were calculated for frequencies in a range from $1 \mathrm{kHz}$ to $100 \mathrm{MHz}$ as shown in Figure 3-2 [79].
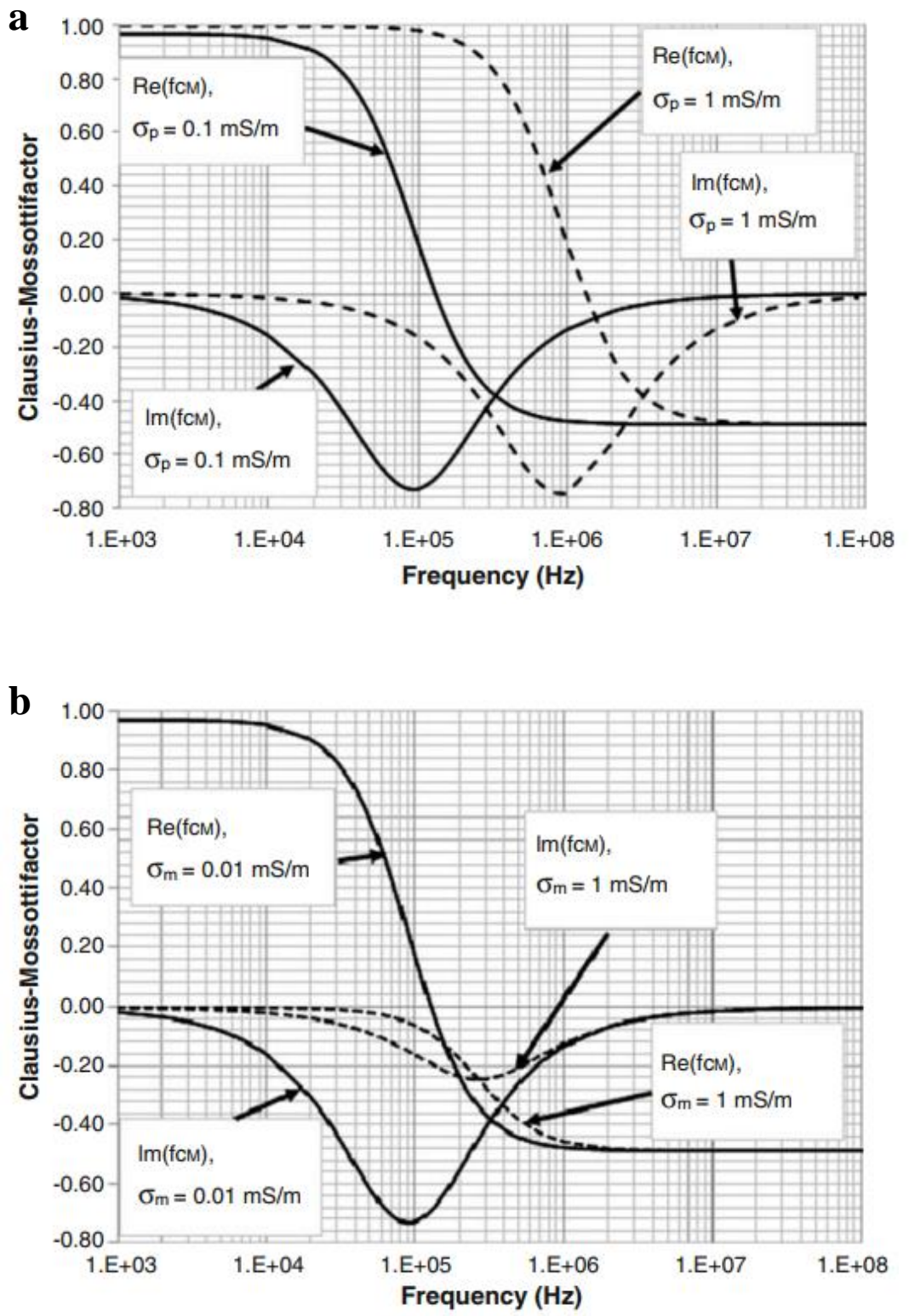

Figure 3-2. (a): CM factor vs. frequency for specific particle conductivities. (b): CM factor vs. frequency for specific medium conductivities.

In Figure 3-2 (a) (case one), we can clearly observe the shift of the DEP spectrum when the conductivity of particles is changing. When the conductivity increases from $0.1 \mathrm{mS} / \mathrm{m}$ to $1 \mathrm{mS} / \mathrm{m}$, 
the crossover frequency of the system shifts to larger frequencies. Therefore, the crossover frequency can be tuned by adjusting the particles conductivity. This can be achieved by a variety of techniques such as surface coating and doping. In Figure 3-2 (b) (case two), significant change in the magnitude of the $K(\omega)$ can be observed when the conductivity of the medium is changing. In contrast, the shift of the spectrum is much smaller comparing with Figure 3-2 (a). When the medium conductivity is $0.01 \mathrm{mS} / \mathrm{m}$, the particle experiences positive DEP force before $100 \mathrm{kHz}$ and negative DEP force after $100 \mathrm{kHz}$; when it is increased to $1 \mathrm{mS} / \mathrm{m}$, the particle experiences negative DEP force for the whole calculated range of frequencies. As a result, adjustment of the conductivity of liquid media can reverse the direction of the DEP force in certain frequency ranges.

\subsection{Other Electrokinetic Effects}

\subsubsection{Electroosmosis}

When a surface is in contact with a polar aqueous medium, due to various mechanisms such as ionization and ion adsorption, the material will acquire a surface electric charge. In the medium, the counterions are attracted to the surface and the co-ions are repelled from the surface. Together with the random thermal motion of the ions, an electric double layer (EDL), is formed, and the distribution of the ions follow a "diffuse" behavior [80].

The schematics of the electrical double layer is shown in Figure 3-3 (a). At the interface between the object and the solution, a single layer of counterions is formed. Due to the hydrated radius of the counterion, the center of the charge cannot directly be attached to the surface. The distance between the surface and the center of the counterion define the inner layer, which is 
called the Stern layer. The thickness of the Stern layer depends on the hydrated radius of the counterions. The plane between the inner layer and outer layer is called the Stern plane, slipping plane or shear plane, and the electrokinetic potential at the shear plane is called the zeta potential

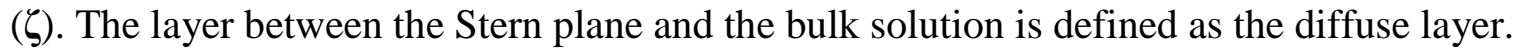

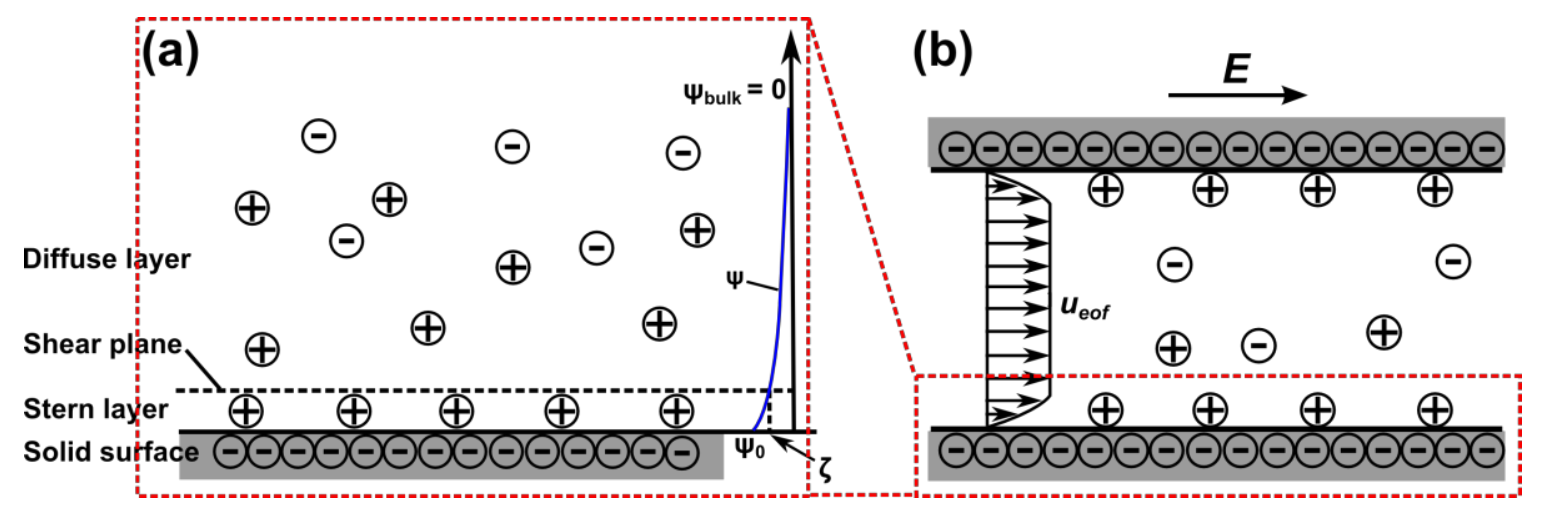

Figure 3-3. (a) Schematics of electrical double layer (EDL). The solid surface is negatively charged and the positive charges are electrostatically attracted to the interface, resulting in the Stern layer. (b) EOF profile in a microfluidic channel. Applied electric field direction: left to right. On the negatively charged wall (gray), positive counter ions are adsorbed, creating an immobile Stern layer. Fluid velocity is uniform throughout the cross section of the channel and drops to zero at the interface (no-slip).

Upon application of an external electric field, redistribution of ions in the vicinity of the interface leads to the increased local charge density, which in turn results in the motion of the fluid, termed electroosmosis (EO). EO is characterized by the bulk fluid motion, therefore the electroosmotic flow (EOF) shows a uniform velocity profile throughout the cross section of the channel and drops rapidly to zero at the solid-liquid interface as shown in Figure 3-3 (b). The electroosmotic velocity $\left(\boldsymbol{u}_{E O}\right)$ is described by the following Smoluchowski Equation [81]:

$$
\boldsymbol{u}_{E O}=\boldsymbol{\mu}_{E O} \boldsymbol{E}=-\frac{\varepsilon \zeta}{\eta} \boldsymbol{E}(\boldsymbol{5})
$$


where $\boldsymbol{\mu}_{E O}$ denotes the electroosmotic mobility, $\boldsymbol{E}$ is the electric field, $\varepsilon$ is the medium permittivity, $\eta$ is the dynamic viscosity of the dispersed medium, and $\zeta$ is the zeta potential. EOF is one of the major electrokinetic flows in microfluidic systems, which is also a parameter to evaluate the surface properties in a microfluidic system.

\subsubsection{Electrophoresis}

Electrophoresis is quite different from dielectrophoresis, though both rely on the interaction of electric fields with particles suspended in media having at least some liquid characteristics. As a brief explanation of this phenomenon, a particle with some net charge is subjected to a homogenous (spatially uniform) electric field exerting an electrostatic force on the particle itself. Due to electrophoresis, the charged particles move along the electric field lines. For instance, a positively charged particle migrates in the cathodic direction, whereas the neutral particle remains stationary [82]. When a particle migrates at a constant velocity under the application of a homogeneous electric field $(\boldsymbol{E})$, it theoretically experiences no net force. The attractive/repulsive force due to the electric field acting on the particle is balanced by the friction force and the retarding force of the diffuse layer of ions being drawn in the opposite direction, and the electrophoretic velocity of the particle $\left(\boldsymbol{u}_{E P}\right)$ is proportional to the applied electric field:

$$
\boldsymbol{u}_{E P}=\boldsymbol{\mu}_{E P} \boldsymbol{E}=\frac{q}{f} \boldsymbol{E}(\mathbf{6})
$$

where $q$ is the particle charge and $f$ is the friction coefficient of the particle. $\boldsymbol{\mu}_{E P}$ is defined as the electrophoretic mobility, and is often interpreted as [83]:

$$
\boldsymbol{\mu}_{E P}=\frac{\varepsilon_{0} \varepsilon_{r} \zeta}{\eta}(\mathbf{7})
$$

where $\eta$ is the dynamic viscosity of the dispersed medium. 


\subsection{Principle of Protein Concentration}

From Equation (3) we can derive that DEP force is proportional to the size of the particle $\left(r^{3}\right), \mathrm{CM}$ factor $(K(\omega))$ and the gradient of the square of the electric field $\left(\nabla \boldsymbol{E}_{r m s}^{2}\right)$. However, it is quite challenging to concentrate protein molecules using classic DEP approaches because of their nanometer sizes and low polarizability, considering the cubic dependence of DEP force on the particle radius. Therefore, Creating a highly enhanced electric field $\left(10^{6}-10^{9} \mathrm{~V} / \mathrm{m}\right)$ and field gradient is generally considered as an effective approach for protein concentration [76, 77]. For the device developed in this research, the constricted channels with abrupt dimensional miniaturization can significantly enhance the electric field and field gradient with over $10^{7}$-fold enhancement resulting a strong DEP force for controlling the protein movement.

When a low-frequency $\mathrm{AC}$ electric field $(<100 \mathrm{kHz})$ is applied, $K(\omega)$ can be approximated using the real conductivities of particle and medium [70, 84]:

$$
K(\omega)=\frac{\sigma_{p}-\sigma_{m}}{\sigma_{p}+2 \sigma_{m}}(\mathbf{8})
$$

Negative DEP $\left(\boldsymbol{F}_{n D E P}\right)$ occurs when the conductivity of protein molecules is smaller than that of the suspending medium, and protein molecules are repelled away from the constricted channel. Since the electrokinetic forces, which are the sum of electroosmosis and electrophoresis, allows for the manipulation of both the particle and the suspending medium, it is possible to realize the trapping and concentration of particles by utilizing electrokinetic and DEP forces. Therefore, to concentrate the protein molecules, in this work a DC electric field was applied and DC electrokinetic forces - electroosmotic force $\left(\boldsymbol{F}_{E O}\right)$ and electrophoretic force $\left(\boldsymbol{F}_{E P}\right)$ execute on proteins to balance $\boldsymbol{F}_{n D E P}$ and results in the concentration. 
Figure 3-4 schematically illustrates protein concentration under the balanced forces of $\boldsymbol{F}_{n D E P}, \boldsymbol{F}_{E O}$ and $\boldsymbol{F}_{E P}$ when a DC-biased AC electric field is applied. For negative charged protein molecules, the directions of $\boldsymbol{F}_{E O}$ and $\boldsymbol{F}_{E P}$ on the same molecule are opposite as shown in Figure 3-4. When both AC and DC electric fields are applied, $\boldsymbol{F}_{n D E P}, \boldsymbol{F}_{E O}$ and $\boldsymbol{F}_{E P}$ act onto the protein molecules simultaneously. On one side of the constricted channel with the cathode for the DC bias, $\boldsymbol{F}_{n D E P}$ and $\boldsymbol{F}_{E O}$ drive the molecules away from the constricted opening, while $\boldsymbol{F}_{E P}$ causes the negatively charged protein molecules to migrate towards the constricted channel. When a force balance is reached as $\boldsymbol{F}_{E P}=\boldsymbol{F}_{n D E P}+\boldsymbol{F}_{E O}$, the protein molecules are accumulated and stabilized in a band region close to the constricted channel as shown in Figure 3-4 [85]. In this case, No concentration will occur on the other side of the constricted channel because no force balance is reached.

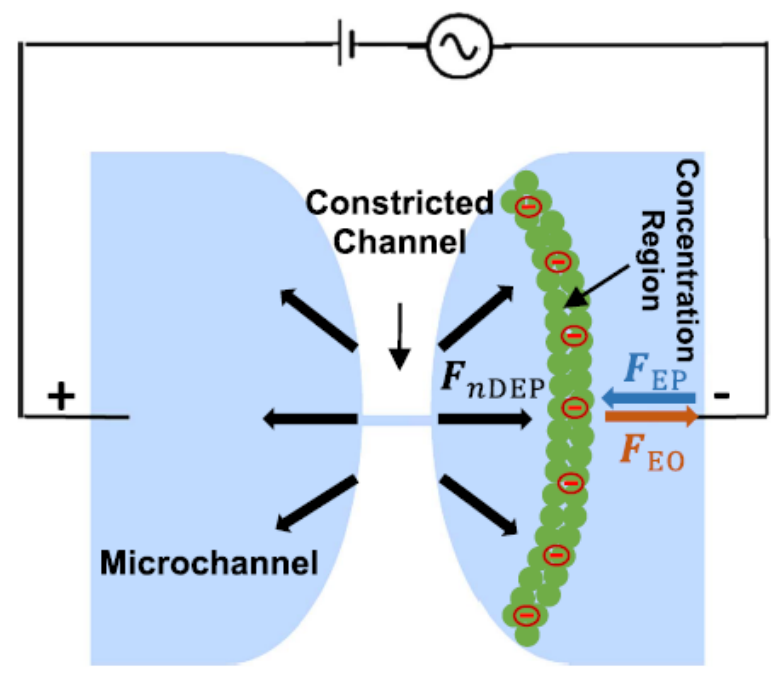

Figure 3-3. Schematic diagram for protein concentration under dielectrophoresis and electrokinetics with an applied DC-biased AC electric field. 


\section{Chapter 4 Materials and Methods}

\subsection{Materials for Device}

In microfluidics, short reaction times, laminar flows and capillary effects are unique phenomena in the microscale and microfluidic systems should be designed in such a way that they take advantage of forces and effects that work on the micro scale. Materials dictate the properties of the microfluidic flow to a large extent [86]. They influence flow rate, capillary pressure, wetting, optical properties, adhesion of biomolecules, cost, and the fabrication methods required. Therefore, the properties of a material are of great importance since they can result in either unique function or problems that do not occur at the macroscale. As a result, the choice of an appropriate material is important to optimizing the function of a microfluidic device.

A variety of materials have been utilized for the fabrication of microfluidic devices. Among these materials, silicon is popular because of well-established micromachining techniques, as well as its mechanical properties, chemical resistance, well characterized processing techniques and the capability for circuitry integration [87]. Glass has also been widely used in microfluidic devices, especially for capillary electrophoresis, due to its optical transparency and ease of electroosmotic flow [88]. Currently, polymers or plastics are widely used for microfluidic devices because of their low manufacturing cost in comparison to glass and silicon and the multiple appropriate properties for performing biochemical analyses. Among all these polymers, a key polymer utilized in microfluidics is Polydimethylsiloxane (PDMS). PDMS is a clear, colorless silicon-based organic polymer, and it is the most popular material for microfluidic research and prototyping due to its ease of fabrication, biocompatibility, nontoxicity, optical transparency and gas permeability [89]. In addition, the micro-scale features can be reproduced 
using PDMS with high precision. Therefore we choose PDMS as the major material to construct our iDEP based microfluidic device.

\subsection{Design}

The PDMS stamp of the microfluidic device was designed with a pattern of three paralleled microridges as shown in Figure 4-1 (a). The microrideges have the same dimensions as $10 \mu \mathrm{m}$ in height, $500 \mu \mathrm{m}$ in width and $15 \mathrm{~mm}$ in length. Molds with different gap distances between two adjacent microrigdes were designed to fit the length of a stretched lambda-DNA ( $\lambda$-DNA), ranging from 10 to $20 \mu \mathrm{m}$. The pattern of electrodes used for applying electric field to the microfluidic device is shown in Figure 4-1 (b). The pair of electrodes has symmetrical structures with a spacing of $500 \mu \mathrm{m}$. Each electrode consists of a long stick with a width of $30 \mu \mathrm{m}$ to apply electric field inside the microchannels and a $5 \mathrm{~mm} \times 5 \mathrm{~mm}$ square pad attached in order to its bottom to provide connection with external power supply. All patterns were sketched by AutoCAD and printed on a high-resolution photomask.

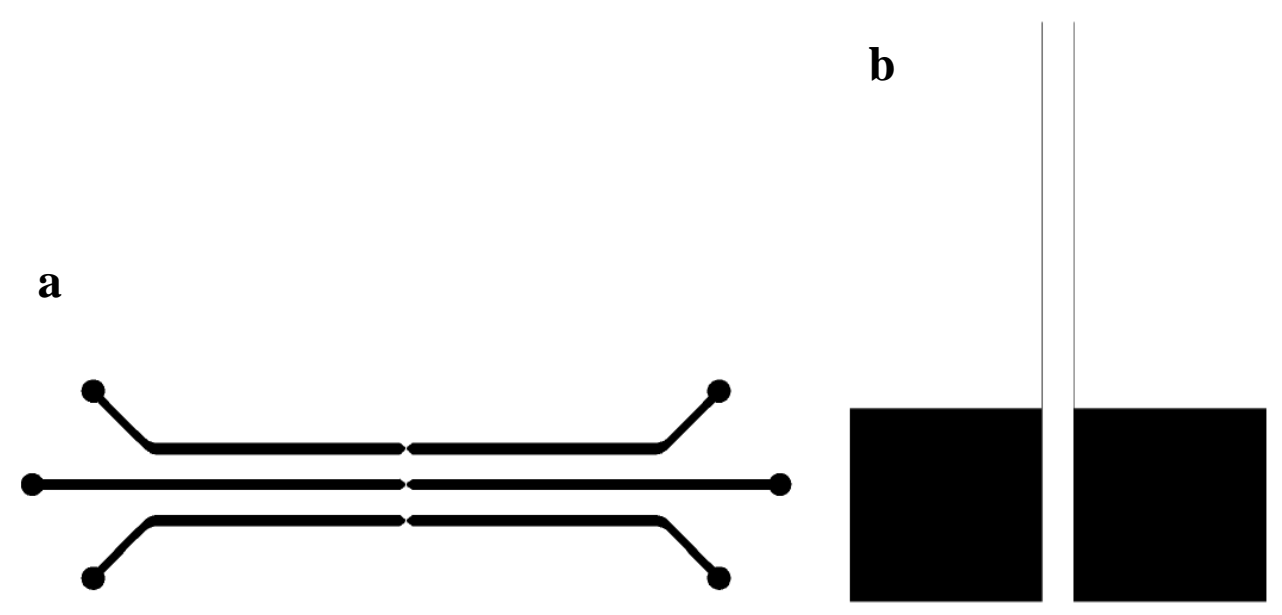

Figure 4-1. (a) Pattern of the PDMS mold of the designed microfluidic device. (b) Pattern of the electrodes for applying electric field. 


\subsection{Fabrication}

Figure 4-2 shows the schematic fabrication process of an iDEP microfluidic device with constricted channels embedded. Preceding this process, master molds of the PDMS stamps were fabricated through standard photolithography. This procedure consisted of utilizing the printed photomask to expose and polymerize a polymer-based photoresist spun cast onto a silicon wafer [90]. Firstly, SU-8 2010 was spin-coated on a silicon wafer at 4000 rpm for 60 seconds. The SU8 coated silicon wafer was baked at $65^{\circ} \mathrm{C}\left(1\right.$ minute) and $95^{\circ} \mathrm{C}$ ( 3 minutes), respectively. Then the SU-8 layer was exposed under UV light. After the post baked at $65^{\circ} \mathrm{C}(1$ minute $)$ and $95^{\circ} \mathrm{C}(3$ minutes), the layer was developed and followed with hard baking at $150^{\circ} \mathrm{C}$ for 30 minutes. The actual thickness of the fabricated layer was measured and confirmed as $10 \mu \mathrm{m}$ using a Profilometer (Tencor Alpha-Step 200). Then the PDMS stamps prepared for further procedures could be fabricated repeatedly with the master molds using standard soft lithography techniques.

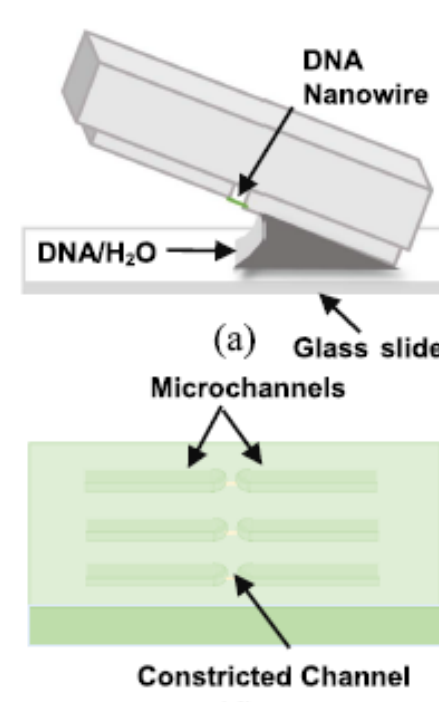

(d)

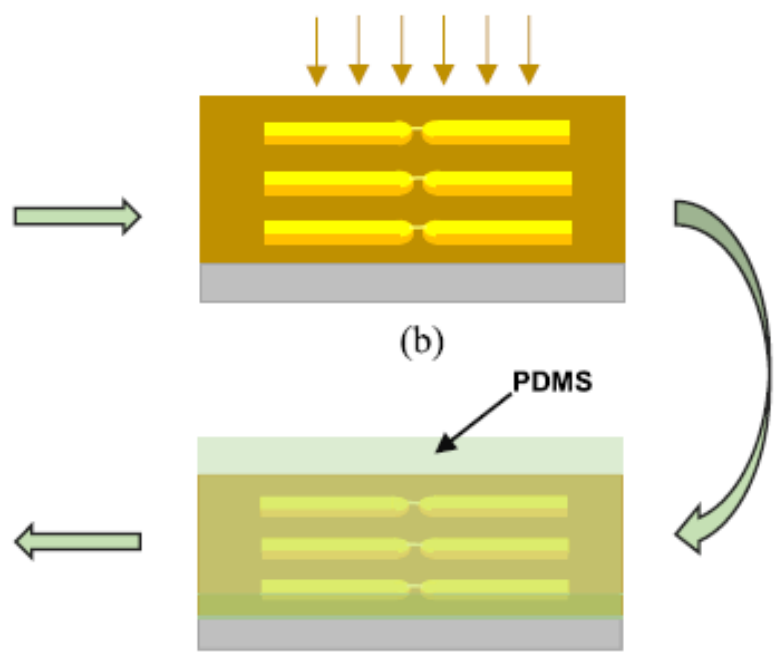

(c)

Figure 4-2. Schematic fabrication process of a PDMS microfluidic device with constricted channels embedded. (a) Stretching DNA nanowire across suspending between two microridges. (b) Sputtered gold-coated DNA nanowires as templates for the constricted channels. (c) PDMS replica cast molding over the gold-coated stamps. (d) Microfluidic device with the embedded constricted channels after removing gold-coated DNA. 
As shown in Figure 4-2, the iDEP device fabrication process includes two major procedures: DNA molecular combing and replica molding. Molecular combing is a class technique to transfer molecules from the solution onto a substrate surface [91, 92] or a patterned feature [33]. First, a patterned PDMS stamp was placed onto a 10 $\mu$ L DNA solution on a glass slide and then slowly peeled off (Figure 4-2 (a)). During this process, the receded solution border caused multiple DNA molecules to stretch along the peeling direction and bundled together by capillary forces to form a nanowire, which bridges the two adjacent microridges after the droplet shrank due to water evaporation and the high surface-to-volume ratio and high surface tension of the aqueous solution. Figure 4-3 illustrates the schematic of the hypothetical process for the formation of DNA nanowires [91]. The DNA solution was prepared by diluting $\lambda$-DNA $(48,502$ bp, $500 \mu \mathrm{g} / \mathrm{mL}$ in $10 \mathrm{mM}$ Tris-HCl/1 mM EDTA, pH 8, New England Biolabs) to $100 \mu \mathrm{g} / \mathrm{mL}$ in a TE buffer solution ( $\mathrm{pH}$ 8, Ambion) and labeled with fluorescent dye (YOYO-1, Invitrogen) at a dye-base pair ratio of 1:5 which was used for microscopic observation.

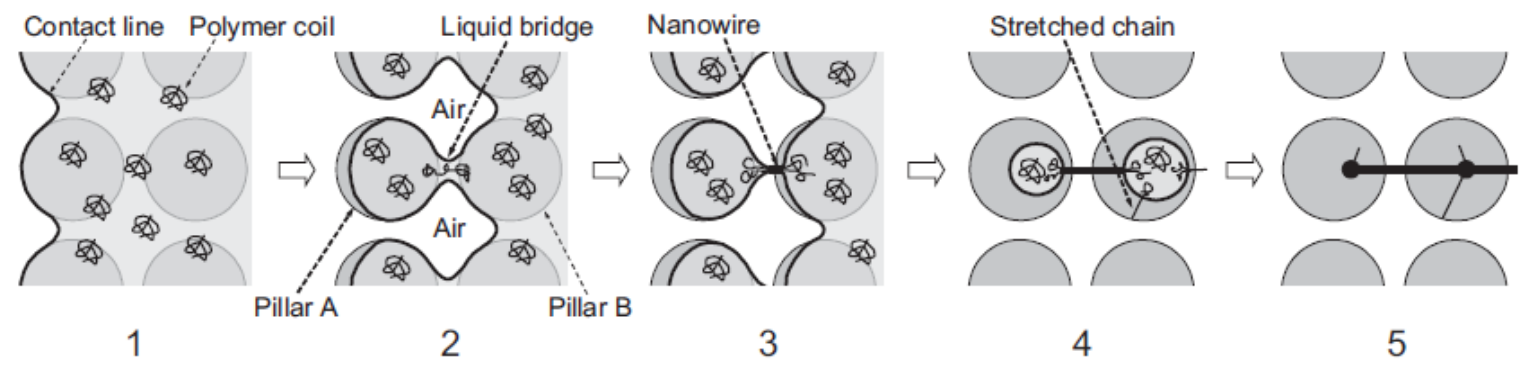

Figure 4-3. Schematic of the hypothetical process for the formation of DNA nanowires.

The stamp with DNA nanowires was then sputtered with gold, generating gold-coated nanowires acting as templates for the constricted channels (Figure 4-2 (b)). Next, the gold-coated stamps were used as the molds for PDMS replica molding (Figure 4-2 (c)). In this step, silicone elastomer base and curing agent (Slygard 184, Dow Chemical) was mixed at a ratio of 10:1, then 
cast onto the gold-coated stamps, and cured in an oven at $60^{\circ} \mathrm{C}$ for 3 hours. The cured PDMS with embedded DNA nanowires were peeled off and soaked in gold etchant (GE-8148, Transene Company Inc.) for 20 minutes, followed by DI water rinsing and nitrogen drying to open the constricted channels and have the microfluidic channels connected (Figure 4-2 (d)). The inlets and outlets for sample solution loading were punched at the end of each microchannel using a $1.5 \mathrm{~mm}$ diameter sharpened puncher. Finally, the device was bonded onto a glass cover with prepatterned electrodes by oxygen plasma treatment.

The electrodes (Ni/Au: 2nm/100nm) were fabricated by E-beam metal deposition, photolithography, and metal etching. During the bonding process, the device was carefully placed onto the glass surface for having the constricted channels accurately positioned between the electrodes.

\subsection{Experimental Setting and Imaging}

The protein solution $(50 \mu \mathrm{g} / \mathrm{mL})$ was prepared by dissolving albumin-fluorescein isothiocyanate conjugate (FITC-albumin) (70 kDa, Sigma-Aldrich) in phosphate buffered saline (PBS) (ATCC) with various conductivities, as shown in Table 4-1 [43, 70]. The solution was incubated for 2 hours at $4^{\circ} \mathrm{C}$. During the experiment, approximately $10 \mu \mathrm{L}$ of solution was slowly injected into the microchannel from the inlet using a syringe via a Tygon tubing (ColeParmer Instrument) under a moderate pressure until the liquid flowed through the narrow constricted channel and filled the downstream microfluidic channel. The entire process took approximately 2-3 minutes. After the protein sample was introduced, a DC biased sinusoidal signal generated by a function generator (CFG250, Tektronix) was applied to the electrodes and adjusted for obtaining optimized concentration results. The amplitudes $\mathrm{V}_{D C}$ for the applied DC 
over the two electrodes were in the range of $0 \mathrm{~V}$ to $2 \mathrm{~V}$, the amplitudes $\mathrm{V}_{P P}$ and the frequencies for the applied AC signal were in the range of $5 \mathrm{~V}$ to $15 \mathrm{~V}$ and $10 \mathrm{~Hz}$ to $100 \mathrm{kHz}$, respectively.

A Nikon Eclipse Ti inverted fluorescence microscope (Nikon Japan) was used for real-time imaging of the concentration results. The microscope is fully automated for imaging multiple positions in sequence, and equipped with long working distance objectives to rapidly acquire high-resolution phase contrast and fluorescent images. The equipped FITC filter, selected for FITC-BSA imaging, allows blue excitation at a wavelength of 475-490 nm and green emission detection at a wavelength of 505-535 nm. For the experiment, a 20× microscope objective was used, and time-lapse fluorescence images were taken every 5 seconds over 2 minutes. Nikon imaging software (NIS-Elements AR 3.2) was used for image processing. Because of the curvature of the protein concentration band, the fluorescence intensities for each result were measured using fifteen ROIs (10×10 micron square) inside the concentration region. The mean values and standard deviations were calculated based on the intensity measurements.

Table 4-1 Medium conductivities of different concentrated PBS buffer and corresponding Clausius-Mossotti (CM) factors of protein particles in the suspending medium. The conductivity of the protein particles $\boldsymbol{\sigma}_{\boldsymbol{p}}$ was taken as $0.0025 \mathrm{~S} / \mathrm{m}$ as a maximum value [70].

\begin{tabular}{|c|c|c|}
\hline PBS Concentration & Conductivity $(\mathrm{S} / \mathrm{m})$ & CM factors \\
\hline $1 \times$ & $1.6 \sim 2$ & -0.499 \\
\hline $0.5 \times$ & $0.8 \sim 1$ & -0.498 \\
\hline $0.2 \times$ & $0.3 \sim 0.4$ & $-0.494 \sim-0.495$ \\
\hline $0.1 \times$ & $0.16 \sim 0.2$ & $-0.488 \sim-0.491$ \\
\hline $0.05 \times$ & $0.08 \sim 0.1$ & $-0.477 \sim-0.481$ \\
\hline
\end{tabular}




\section{Chapter 5 Results and Discussion}

\subsection{Fabrication of Constricted Channel within Microfluidic Device}

The key steps for fabricating the constricted channel together with microfluidic channels are shown in Figure 5-1, including DNA nanowire bridging between two microridges (Figure 5-1 (a)), gold-coated DNA nanowire (Figure 5-1 (b)), and a constricted channel embedded in PDMS and connected to microchannels (Figure 5-1 (c)). The reason of the darkness shown on PDMS (Figure 5-1 (b)) was because the existing gold layer on the PDMS surface blocked the light transmission. As shown in Figure 5-1 (d), the device with embedded constricted channels was then bonded onto a glass cover with pre-patterned electrodes. DNA combing and molding fabrication shown here is simple for creating submicron-scaled constricted channel without relying on e-beam lithography technique. The constricted channel was directly created within the microfluidic channels (Figure 5-1 (e)), avoiding multiple complicated fabrication procedures generally required for generating similar channel structures. The sputtered gold coating provides flexibility in controlling the dimensions (submicron down to nanometer sizes) of the constricted channel. Local electric field near the constricted channel is significantly enhanced. The width of constricted channels obtained in our devices is close to $1 \mu \mathrm{m}$ as measured using the microscope equipped Nikon imaging software.

The field-focusing factor is determined by the dimensional ratio of the cross section at the microchannel where the driving electrodes sit to that at the constricted channel. The method for calculating the enhancement factor of DEP force has been published by Chou's group earlier, and their simulation results demonstrated that the enhanced electrical field $\left(\boldsymbol{E}^{2}\right)$ was in the same order with the field gradient $\left(\nabla \boldsymbol{E}^{2}\right)[75,76]$. In the fabricated device, the field-focusing factor is 
$\sim 5000$, leading to an approximate enhancement factor of $\sim 2.5 \times 10^{7}$ for the dielectrophoretic force at the constriction region. The enhancement factor is in the similar range as previously reported for dielectrophoresis based protein concentration [93, 94].

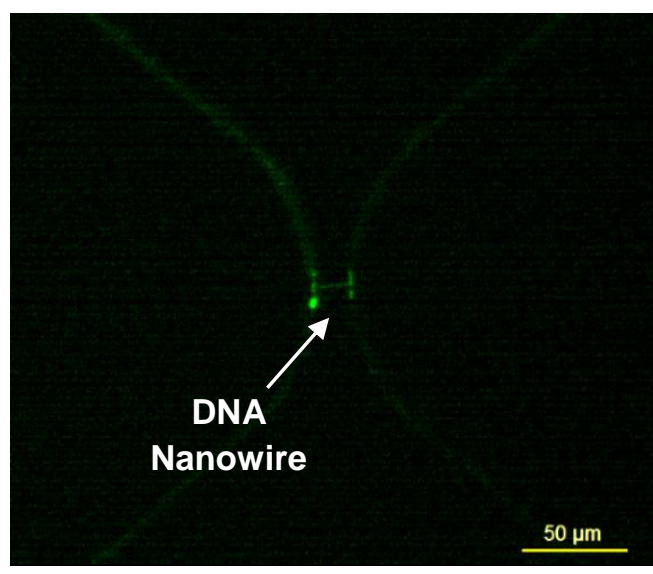

(a)

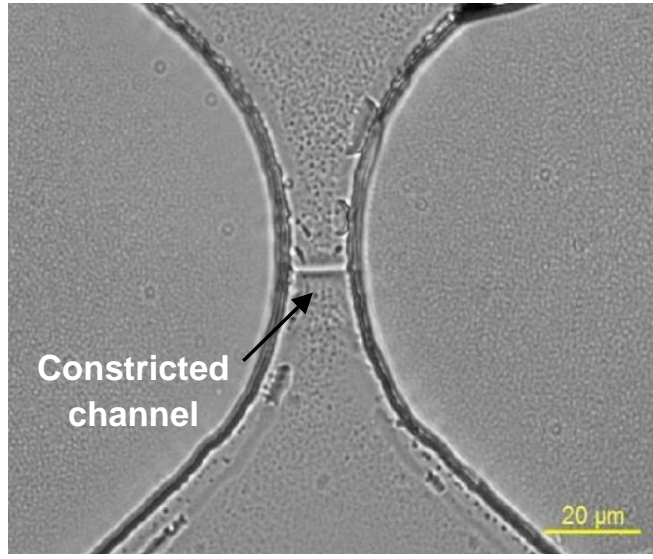

(c)

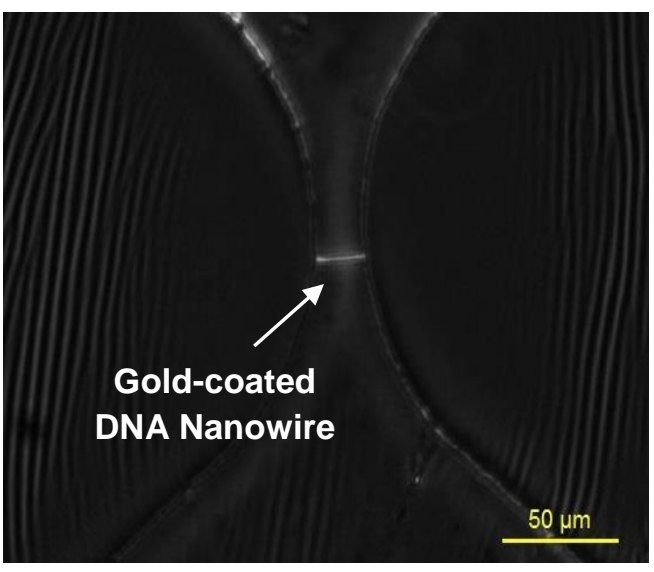

(b)

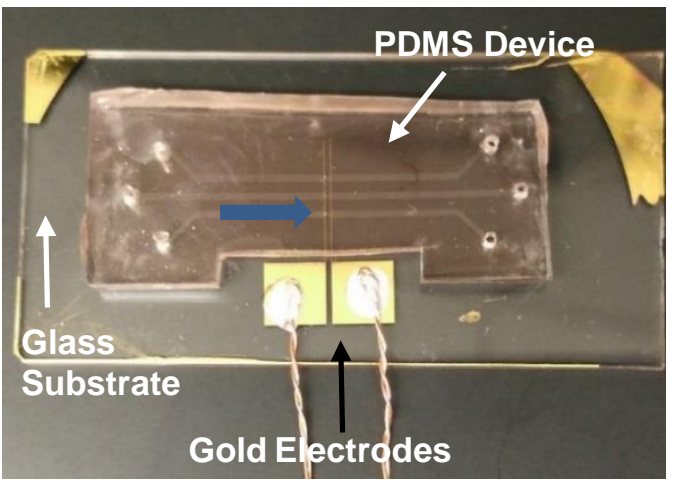

(d)

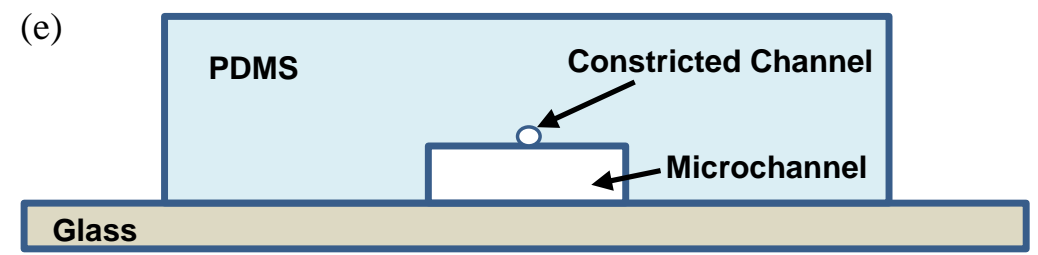

Figure 5-1. (a) Fluorescence image of YOYO-1 labeled DNA nanowires across suspending between two microridges. (b) Representative image shows gold-coated DNA nanowires connecting two microridges. (c) Representative image shows the constricted channel embedded within PDMS and connecting two microchannels after replica molding and gold etching. (d) An assembled microfluidic device for protein concentration. (e) Schematic diagram of the crosssection at one end of the microchannel viewed from the direction pointed by the bold arrow shown in (d). The scale does not reflect the actual size and geometry of the microstructure. 


\subsection{Effects of Electric Field Parameters}

The applied electrical fields can dramatically affect the concentration results, including the speed of protein enrichment, the concentration intensities, and the position of the concentrated molecules. All of those can be manipulated by the combination of applied AC amplitudes, DC biases, AC frequencies, and dimensional ratio between the microchannel and the constricted channel. To study electrical parameters' effects on the protein concentration, different scenarios were performed by varying either the applied AC amplitudes, AC frequencies, or DC biases. FITC-albumin with an initial concentration of $50 \mu \mathrm{g} / \mathrm{ml}$ and suspending medium conductivity of $0.08 \sim 0.1 \mathrm{~S} / \mathrm{m}$ was used for this study.

\subsubsection{AC Amplitude}

First, the combination effects of DC bias and AC amplitudes (with fixed AC frequency at 1 $\mathrm{kHz}$ ) were demonstrated for the protein concentration as shown in Figure 5-2. Each image shows the concentration results at 100 seconds after the electric fields were applied. Without DC biasing, no concentration effect was observed under $\mathrm{AC}$ electric field $\left(\mathrm{V}_{P P}: 5 \mathrm{~V}\right.$, frequency: 1 $\mathrm{kHz}$ ) as shown in Figure 5-2 (a). This is because no electrokinetic forces were presented and the dominant $\boldsymbol{F}_{n D E P}$ caused the protein molecular depleted away from the constriction region.

When DC biased AC electric field was applied, with the significantly enhanced electric field gradient, $\boldsymbol{F}_{n D E P}$ was quickly dominant in the region close to the constricted channel comparing to the DC electrokinetics forces $\boldsymbol{F}_{E P}$ and $\boldsymbol{F}_{E O}$, thus the depletion of protein molecules

was first observed because the molecules were repelled by the dominant $\boldsymbol{F}_{n D E P}$ against $\boldsymbol{F}_{E P}$ in the opposite direction. 


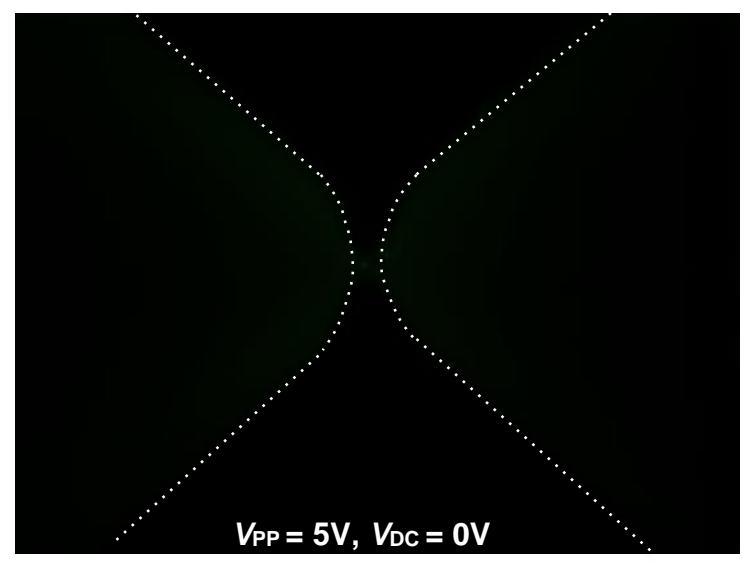

(a)

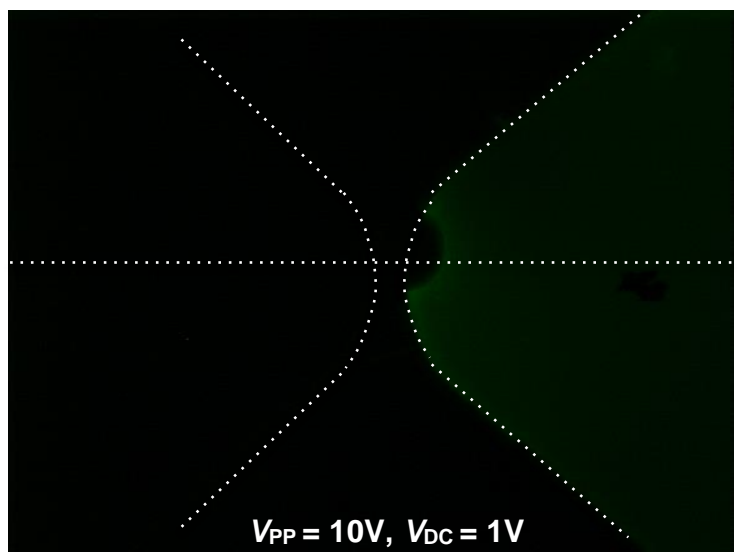

(c)

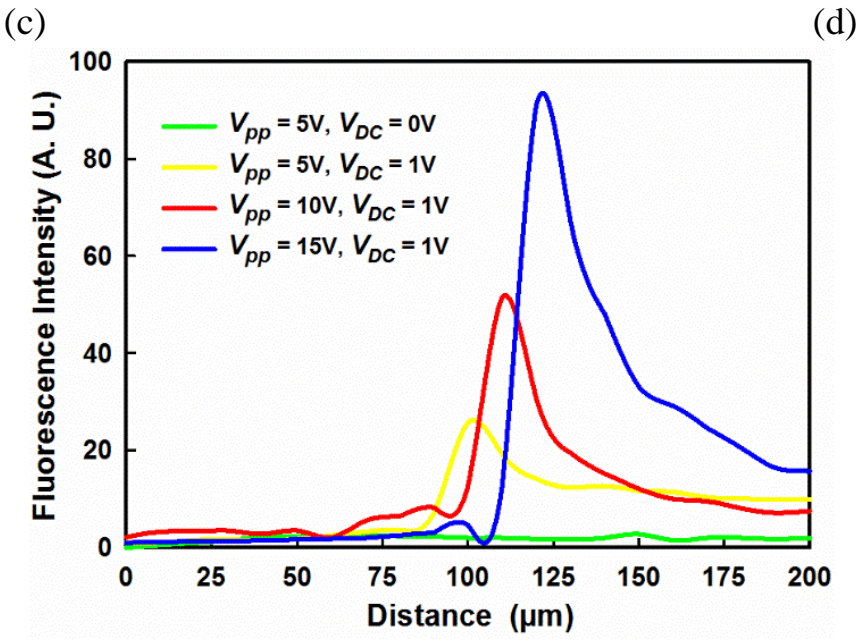

(e)

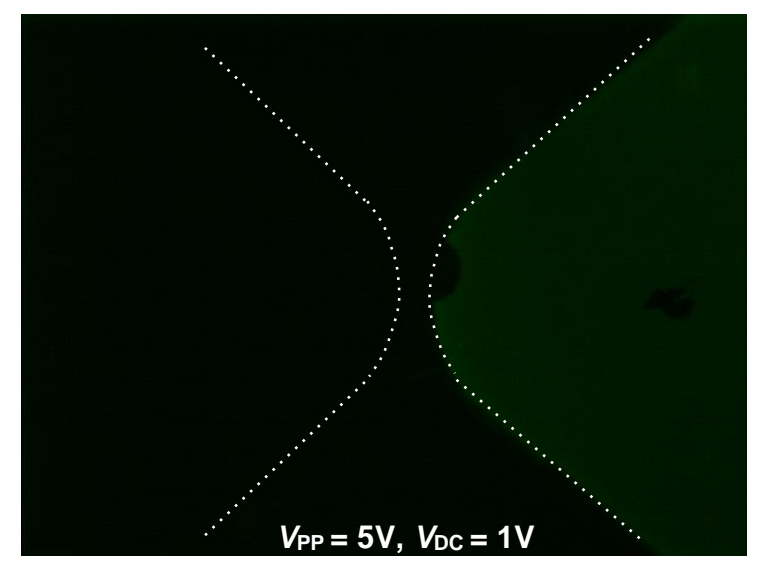

(b)

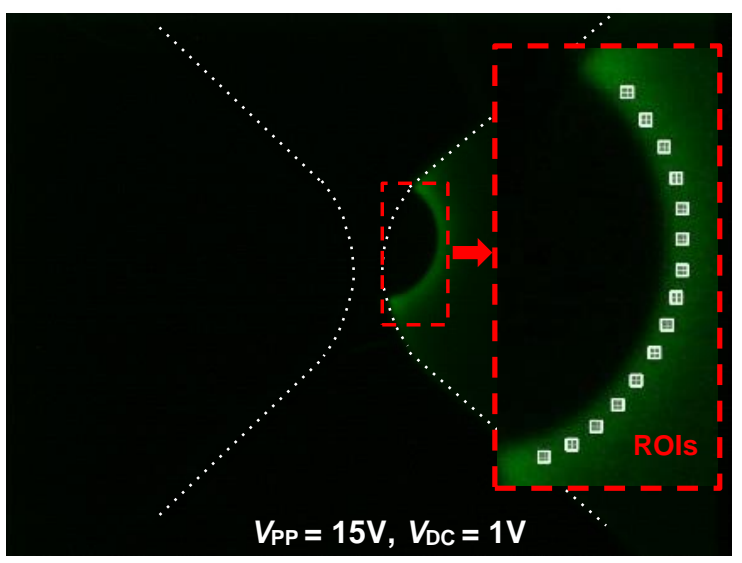

(d)

Figure 5-2. Fluorescence images show FITC-BSA concentration results when an AC electric field $(1 \mathrm{kHz})$ was applied for 100 seconds with the condition of (a) $\mathrm{V}_{P P}=5 \mathrm{~V}, \mathrm{~V}_{D C}=0 \mathrm{~V}$, (b) $\mathrm{V}_{P P}=5 \mathrm{~V}, \mathrm{~V}_{D C}=1 \mathrm{~V}$, (c) $\mathrm{V}_{P P}=10 \mathrm{~V}, \mathrm{~V}_{D C}=1 \mathrm{~V}$, (d) $\mathrm{V}_{P P}=15 \mathrm{~V}, \mathrm{~V}_{D C}=1 \mathrm{~V}$, respectively. (e) Fluorescence intensity under different electric fields conditions. The $\mathrm{X}$-axis indicates the horizontal positions across the image plane from left end to right end, and the intensity values were measured along the direction of constricted channel at the middle vertical height for every condition, as illustrated by the flat dotted line shown in figure (c). 
For nanometer-sized particles within a salt solution, the electrophoresis effect is much greater than the electroosmosis because electrophoresis mobility significantly exceeds electroosmosis mobility. Gradually, with the force balance reached, protein enrichment was achieved and stabilized in an arc-shape band near one side of the constricted channel, as demonstrated in Figure 3-4 and shown in Figure 5-2.

For more details, when a DC (1V) biased AC electric field ( $\mathrm{V}_{\mathrm{PP}}: 5 \mathrm{~V}$, frequency $\left.1 \mathrm{kHz}\right)$ was applied as shown in Figure 5-2 (b), the electrophoretic force $F_{E P}$ caused the molecules to flow back to the constriction region, and under the combination effects of $\boldsymbol{F}_{E P}, \boldsymbol{F}_{E O}$ and $\boldsymbol{F}_{n D E P}$, the molecules were gradually accumulated at one side of the constricted channel till a stable concentration region formed under the force balance. To further enhance the concentration responses, the applied AC amplitudes $\mathrm{V}_{P P}$ were increased to $10 \mathrm{~V}$ (Figure 5-2 (c)) and 15V (Figure 5-2 (d)), respectively, while the DC bias was kept the same. As shown in Figure 5-2 (c) and 5-2 (d), the concentration band kept moving further away from the constricted channel because of the increase in $\boldsymbol{F}_{n D E P}$ caused by the increasing of AC amplitudes. The concentration was stabilized and further enhanced for trapping more protein molecules until the force balance was established between $\boldsymbol{F}_{n D E P}$ and DC electrokinetic forces $\boldsymbol{F}_{E P}$ and $\boldsymbol{F}_{E O}$. The quantified fluorescence intensities under different electric fields conditions are shown in Table 5-1. For statistical analysis, data was presented as the mean \pm standard deviation (SD) and the fluorescence intensity measurement for each individual experiment was performed 15 times.

On the other side of the constricted channel, no protein concentration occurred because all three forces were in the same direction at this side and no force balance can occur. Hence, with the application of a DC-biased AC electric field, protein concentration can be effectively achieved at one side of the constricted channel towards the cathode of DC bias. 
Table 5-1 Average values and standard deviations of fluorescence intensity values measured from 15 ROIs along the concentration band as shown in Figure 5-2 (d) indicated using an enlarged image. The same measurement was also applied for Figure 5-2 (b) and (c). The fluorescence intensity data was measured using Nikon imaging software (NIS-Elements AR 3.2).

\begin{tabular}{|c|c|c|}
\hline \multicolumn{2}{|c|}{ Electric Field } & \multirow{2}{*}{$\begin{array}{l}\text { Fluorescence Intensities (Arb.Units) } \\
\quad(\text { Mean } \pm \text { Standard Deviation) }\end{array}$} \\
\hline $\mathrm{AC}(f=1 \mathrm{kHz})$ & DC & \\
\hline $\mathrm{V}_{\mathrm{pp}}=5 \mathrm{~V}$ & $0 \mathrm{~V}$ & $1.92 \pm 0.48$ \\
\hline $\mathrm{V}_{\mathrm{pp}}=5 \mathrm{~V}$ & $1 \mathrm{~V}$ & $25.3 \pm 1.16$ \\
\hline $\mathrm{V}_{\mathrm{pp}}=10 \mathrm{~V}$ & $1 \mathrm{~V}$ & $51.6 \pm 1.57$ \\
\hline $\mathrm{V}_{\mathrm{pp}}=15 \mathrm{~V}$ & $1 \mathrm{~V}$ & $90.4 \pm 2.28$ \\
\hline
\end{tabular}

\subsubsection{Bias}

As shown in Figure 5-3, time-lapse fluorescence images were taken under the condition of varied amplitudes of the DC bias with fixed AC electric field $\left(\mathrm{V}_{P P}: 15 \mathrm{~V}\right.$, frequency $\left.10 \mathrm{~Hz}\right)$. When the concentration was stabilized after the application of electric fields for 100 seconds, the intensity of the concentration band under lower DC electric field (1V) (Figure 5-3 (c)) was low comparing to the intensity under a higher DC electric field (2V) (Figure 5-3 (f)). Moreover, the concentration band shifted towards the constricted channel under a higher DC bias as compared from the left column of images (Figure 5-3 (a)-(c)) and the right column of images (Figure 5-3 (c)-(f)). When the amplitude of the applied DC bias was increased, $\boldsymbol{F}_{E P}$ exerted on the concentrated molecules was also increased, thus the pre-established force balance was temporarily broken $\left(\boldsymbol{F}_{E P}>\boldsymbol{F}_{n D E P}+\boldsymbol{F}_{E O}\right)$, resulting in the concentrated molecules moving towards the constricted channel. With the increasing $\boldsymbol{F}_{n D E P}$ near the constricted channel, a new force balance re-established and concentration was further enhanced as compared and shown in the left and right columns of images in Figure 5-3. 


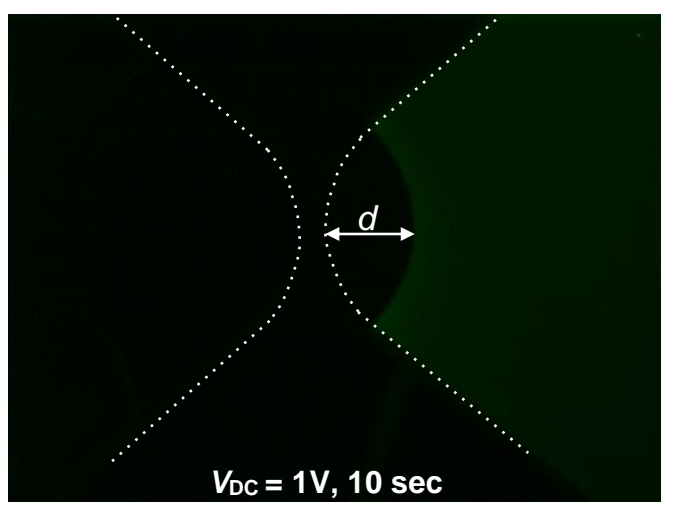

(a)

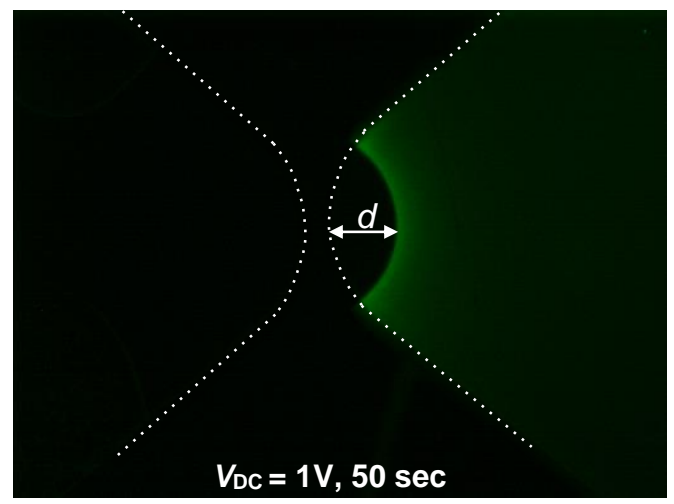

(b)

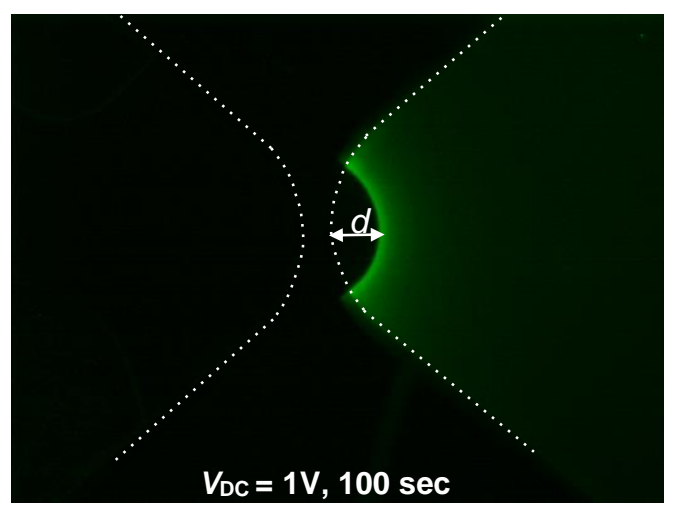

(c)

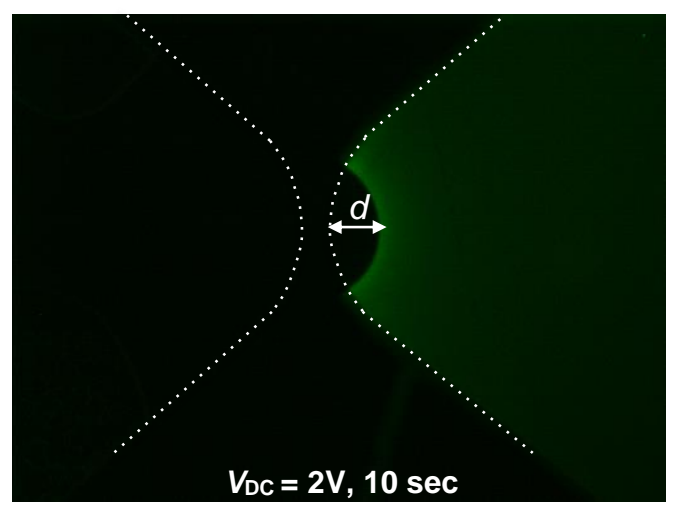

(d)

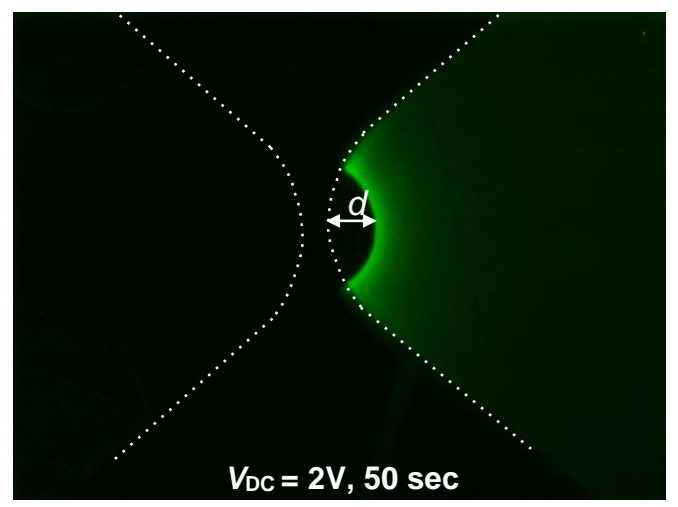

(e)

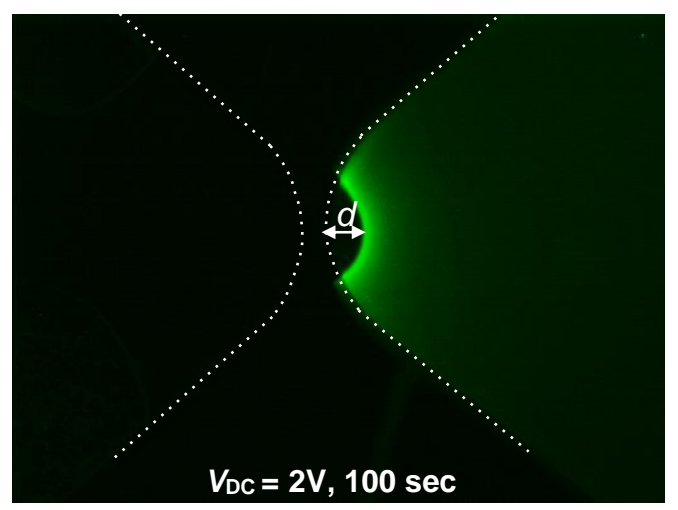

(f) 


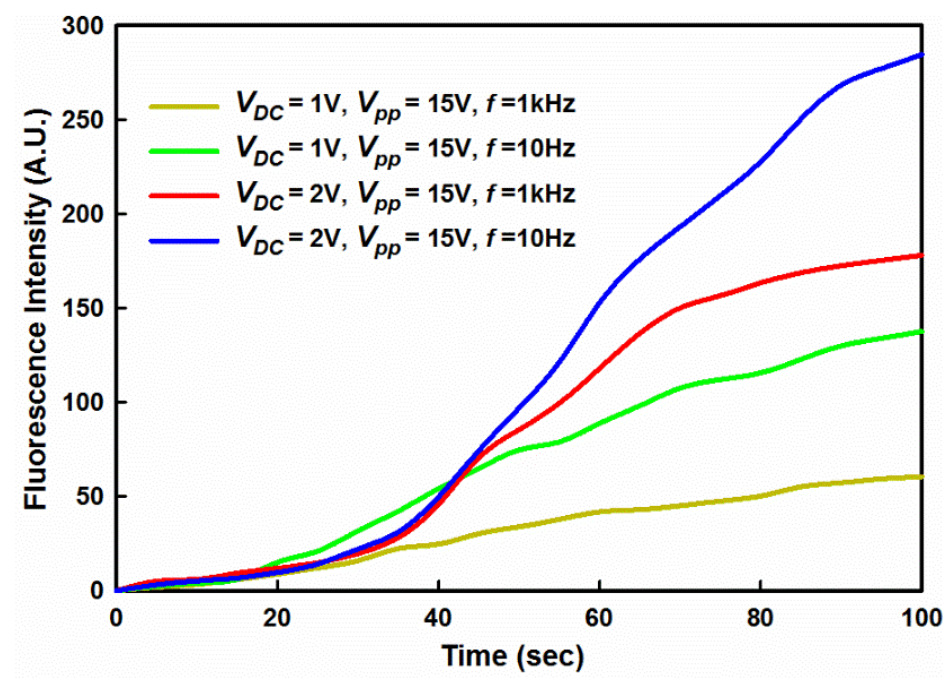

$(\mathrm{g})$

Figure 5-3. Representative FITC-BSA concentration results under AC electric field $\left(\mathrm{V}_{P P}=15 \mathrm{~V}\right.$, frequency $=10 \mathrm{~Hz}$ ) with different DC biases. (a) (c) Fluorescence images show the results under AC electric field with 1V DC bias after 10 seconds, 50 seconds and 100 seconds, respectively; (d) (f) Fluorescence images show the results under AC electric filed with 2V DC bias after 10 seconds, 50 seconds and 100 seconds, respectively; (g) Fluorescence intensities of the concentration over time under $\mathrm{AC}$ electric fields $\left(\mathrm{V}_{P P}=15 \mathrm{~V}\right)$ at frequencies of $10 \mathrm{~Hz}$ and $1 \mathrm{kHz}$ applied with $1 \mathrm{~V}$ and $2 \mathrm{~V}$ DC biases, respectively.

\subsubsection{AC Frequency}

Additionally, the effects of AC frequencies were studied. As shown in Figure 5-4, the concentration band shifted towards to the constricted channel when the AC frequencies were decreased from $1 \mathrm{kHz}$ to $10 \mathrm{~Hz}$. This is because the electrokinetic mobility of the particles is frequency dependent, associating with phase lags and the effects of particle inertia [95]. At a low $\mathrm{AC}$ frequency, the inertial force executed on the particles can be negligible and the particles move in phase with the AC electric field at the same speed as in a DC electric field. At a higher frequency $(\sim \mathrm{kHz})$, the effects of particle inertia become significant, causing the speed of the particles to decline and their motions to lag behind the applied electric field. In such a situation, 
the particle's electrophoretic mobility $\boldsymbol{\mu}_{E P}$ decreases, leading to a weaker $\boldsymbol{F}_{E P}$, thus a reduced $\boldsymbol{F}_{n D E P}$ is necessary to re-establish the force balance.

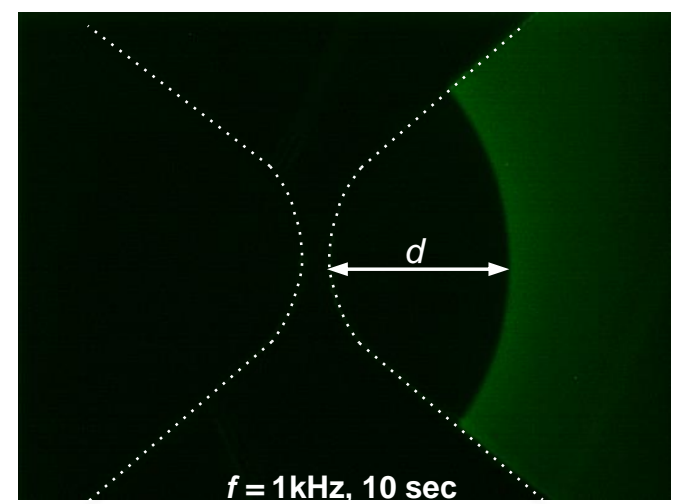

(a)

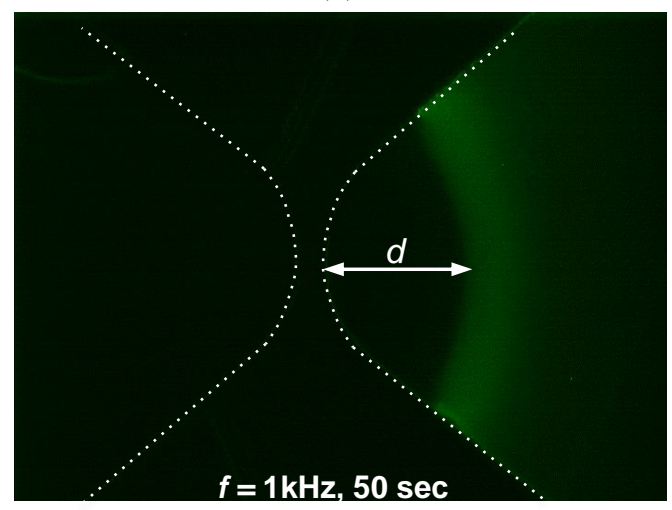

(b)

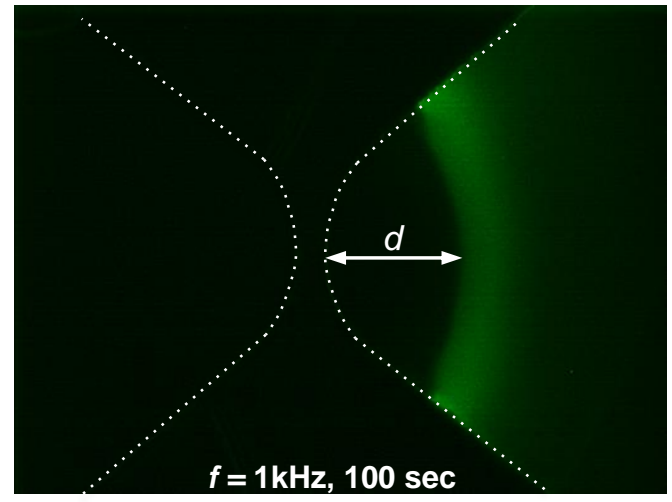

(c)

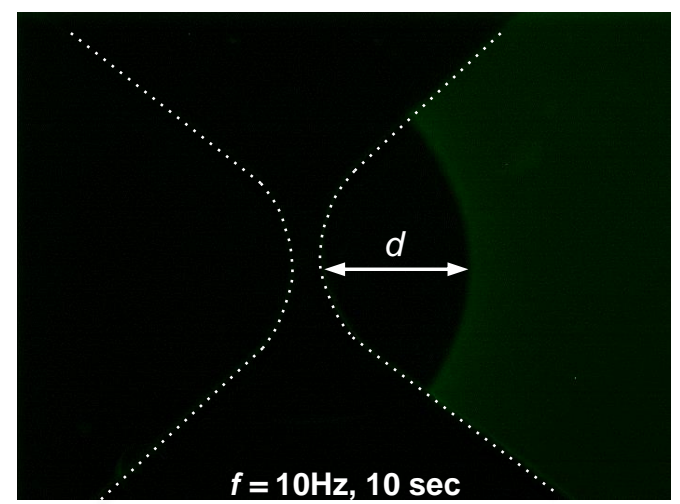

(d)

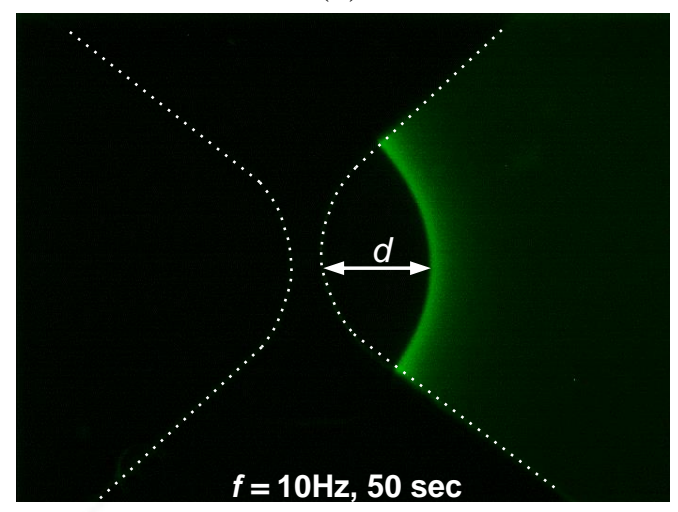

(e)

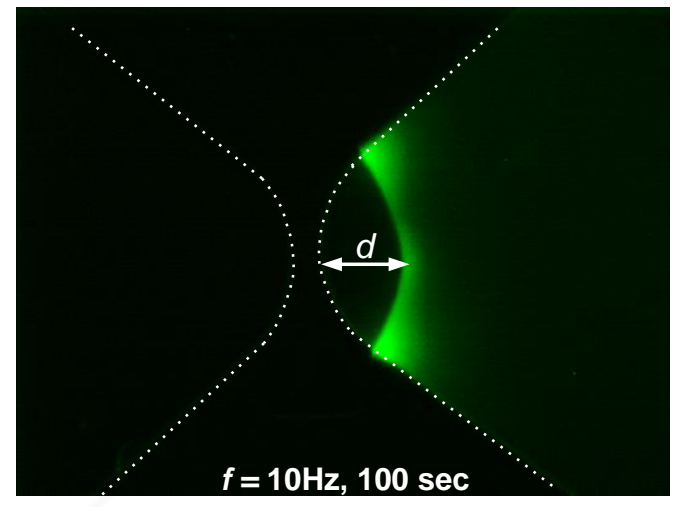

(f)

Figure 5-4. Representative FITC-BSA concentration results under different AC frequencies with the fixed $\mathrm{AC}$ amplitude $\left(\mathrm{V}_{P P}=15 \mathrm{~V}\right)$ and $\mathrm{DC}$ bias $(2 \mathrm{~V})$. (a) (c) Fluorescence images show the results under $1 \mathrm{kHz}$ frequency after 10 seconds, 50 seconds and 100 seconds, respectively. (d) (f) Fluorescence images show the results under $10 \mathrm{~Hz}$ frequency after 10 seconds, 50 seconds and 100 seconds, respectively. 
Based on the experimental results shown in Figure 5-2, Figure 5-3 and Figure 5-4, the average distance $d$ of the concentration band away from the constricted channel, was measured and shown in Table 5-2 with varied AC amplitudes, AC frequencies and DC biases. The results show that the applied electric fields can be adjusted to manipulate the concentrated molecules and their concentration region. The concentration is micrometers away from the local significantly enhanced electric field at the constriction. This also provides flexibility for integrating other sensing units within the device.

Table 5-2 Average distances between the concentration band and the constricted channel with varied AC amplitudes, AC frequencies and DC biases applied.

\begin{tabular}{|c|c|c|c|}
\hline $\begin{array}{c}\text { DC Bias } \\
(\mathbf{V})\end{array}$ & $\begin{array}{c}\text { AC } \\
\text { Amplitude (V) }\end{array}$ & $\begin{array}{c}\text { AC Frequency } \\
(\mathbf{H z})\end{array}$ & $\begin{array}{c}\text { Distance }(\boldsymbol{d}) \\
\text { (Mean } \mathbf{\pm} \text { Standard Deviation) }(\boldsymbol{\mu m})\end{array}$ \\
\hline 1 & 5 & 1000 & $100.4 \pm 6.2$ \\
\hline 1 & 10 & 1000 & $113.9 \pm 5.5$ \\
\hline 1 & 15 & 1000 & $134.6 \pm 7.8$ \\
\hline 1 & 15 & 10 & $83.2 \pm 5.6$ \\
\hline 2 & 15 & 1000 & $45.7 \pm 6.1$ \\
\hline 2 & 15 & 10 & $23.8 \pm 1.8$ \\
\hline
\end{tabular}

\subsection{Effects of Medium Conductivity}

To investigate optimum conditions for protein concentration, the effects of medium conductivities on concentration results were conducted under the same electrical field strength $\left(V_{P P}=15 \mathrm{~V}, V_{D C}=2 \mathrm{~V}\right)$ with varying $\mathrm{AC}$ frequencies. The same concentrated FITC-BSA solutions $(50 \mu \mathrm{g} / \mathrm{ml})$ were prepared in PBS with five different concentrations $(0.05 \times, 0.1 \times, 0.2 \times, 0.5 \times$ and 1×), which were corresponding to five different conductivities presented in Table 4-1. The AC 
frequencies varied from $10 \mathrm{~Hz}$ to $100 \mathrm{kHz}$ were applied to each medium condition, respectively.

Figure 5-5 shows the concentration results under the different medium conductivities with varying $\mathrm{AC}$ frequencies.

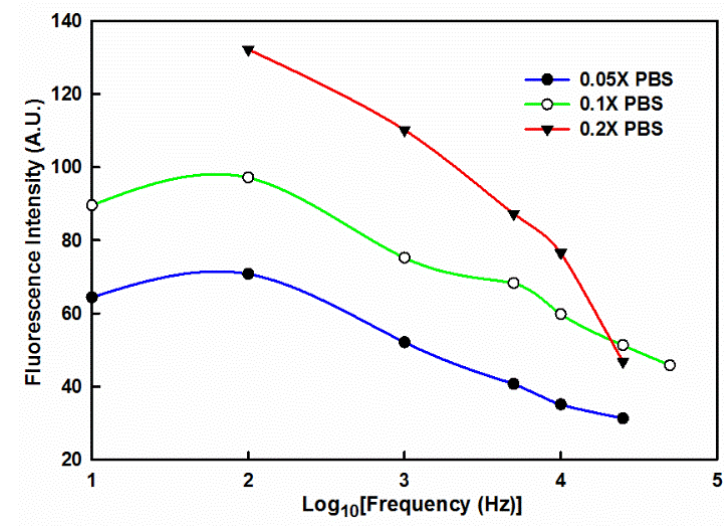

(a)

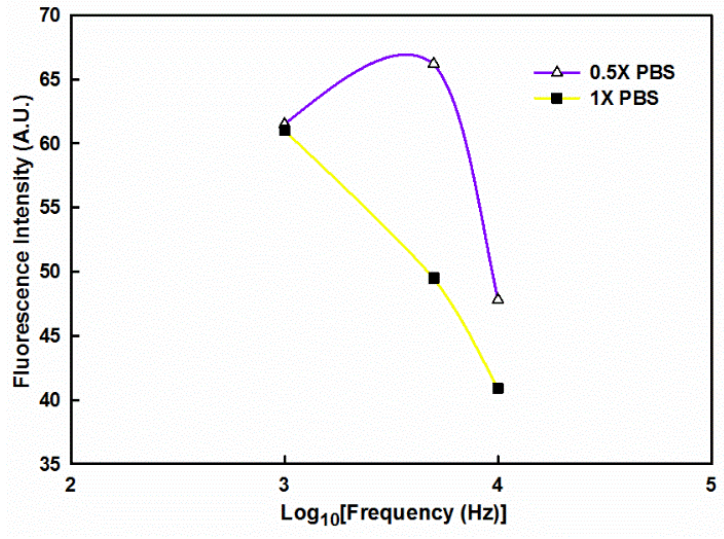

(b)

Figure 5-5. FITC-BSA concentration results obtained under a combination of varying medium conductivities and $\mathrm{AC}$ frequencies with the fixed $\mathrm{AC}$ amplitude $\left(\mathrm{V}_{P P}=15 \mathrm{~V}\right)$ and $\mathrm{DC}$ bias (2V). (a) Results obtained under $0.05 \times, 0.1 \times$, and $0.2 \times$ PBS; (b) Results obtained under $0.5 \times$ and $1 \times$ PBS.

For lower media conductivities $(0.05 \times, 0.1 \times$, and $0.2 \times$ PBS $)$ shown in Figure 5-5 (a), molecules enrichment was well observed at frequencies below $25 \mathrm{kHz}$. For higher media conductivities ( $0.5 \times$ and $1 \times$ PBS) shown in Figure 5-5 (b), molecules enrichment was observed at intermediate frequencies $(1 \mathrm{kHz} \sim 10 \mathrm{kHz})$, however with lower fluorescence intensities compared to the results for lower medium conductivities at the same frequency. As observed, the concentration intensities decreased with increasing frequency. This is in agreement with and further proves the results presented in Figure 5-3 and Figure 5-4.

Furthermore, at lower medium conductivities (less than 0.2× PBS) shown in Figure 5-5 (a), the concentration intensities increase with the increasing of medium conductivities under the same applied frequencies. As stated earlier, CM factors can be approximated using the real conductivities of particle and medium when the applied AC frequencies are lower than $100 \mathrm{kHz}$. 
The calculated values of the CM factors for protein molecules in each different media are listed in Table 4-1. The magnitude of the negative CM factors increased by increasing the medium conductivities, resulting in the increasing of negative dielectrophoretic force $\boldsymbol{F}_{n D E P}$ near the constricted channel. When a new force balance between the $\boldsymbol{F}_{n D E P}$ and DC electrokinetic forces $\boldsymbol{F}_{E P}$ and $\boldsymbol{F}_{E O}$ was established, protein enrichment was further enhanced comparing to that at lower medium conductivity. At higher medium conductivities $(0.5 \times$ and $1 \times$ PBS $)$ shown in Figure 5-5 (b), with the strength of $\boldsymbol{F}_{n D E P}$ kept increasing, the force balance cannot be fully established and fewer protein molecules can be concentrated, showing a reduced intensity under the same applied frequencies.

No successful concentration was observed at higher frequencies $(50 \mathrm{kHz} 100 \mathrm{kHz})$ under any of the media conductivities. The protein molecules were quickly driven away from the constricted channel. As discussed earlier, balance of forces cannot be achieved because of the dramatically decreased DC electrokinetic force $\boldsymbol{F}_{E P}$ at high frequencies.

The results provide information on how to improve the performance of constriction-based dielectrophoresis presented in this work for achieving an optimal concentration results by manipulating operating conditions regarding the medium conductivities and applied frequencies.

\subsection{Measurement Sensitivity of Protein Concentration}

Another group of experiments were carried out to further explore the measurement sensitivity of the protein concentration effect based on the developed device and proposed method. Figure 5-6 demonstrates the representative protein concentration results under the same electrical field condition using FITC-BSA samples with different initial concentrations, ranging from $100 \mathrm{ng} / \mathrm{mL}$ to $100 \mu \mathrm{g} / \mathrm{mL}$. As indicated by Table 4-1, the conductivity of the FITC-BSA 
particles $(0.0025 \mathrm{~S} / \mathrm{m})$ is much smaller than the medium conductivity with even the lowest PBS concentration $(0.08 \sim 0.1 \mathrm{~S} / \mathrm{m})$, which suggests that changes in the sample's initial concentration would not necessarily affect the crucial parameter of medium conductivity and thus could be studied separately. The values of the fluorescence intensity within the enhanced concentration region before and after the protein concentration process were measured from each result shown in Figure 5-6 (a) (f) and displayed in the curves plotted in 5-6 (g), which imply that with the increasing of the sample's initial concentration, the enhanced concentration of the DEP-focused protein molecules also increased in an approximately linear trend. This result indicates that this device could provide and maintain a reasonably constant measurement sensitivity of the protein concentration responses when the samples with various initial concentrations were applied, verifying its versatility over a wide range of sample conditions.

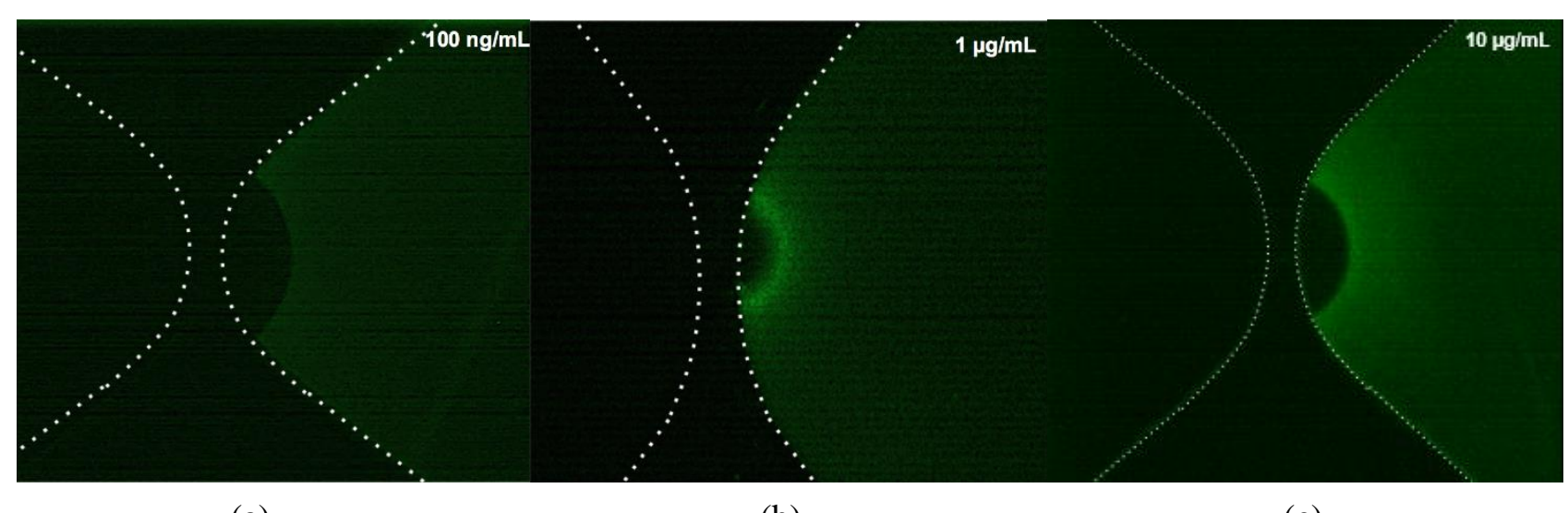

(a)

(b)

(c)

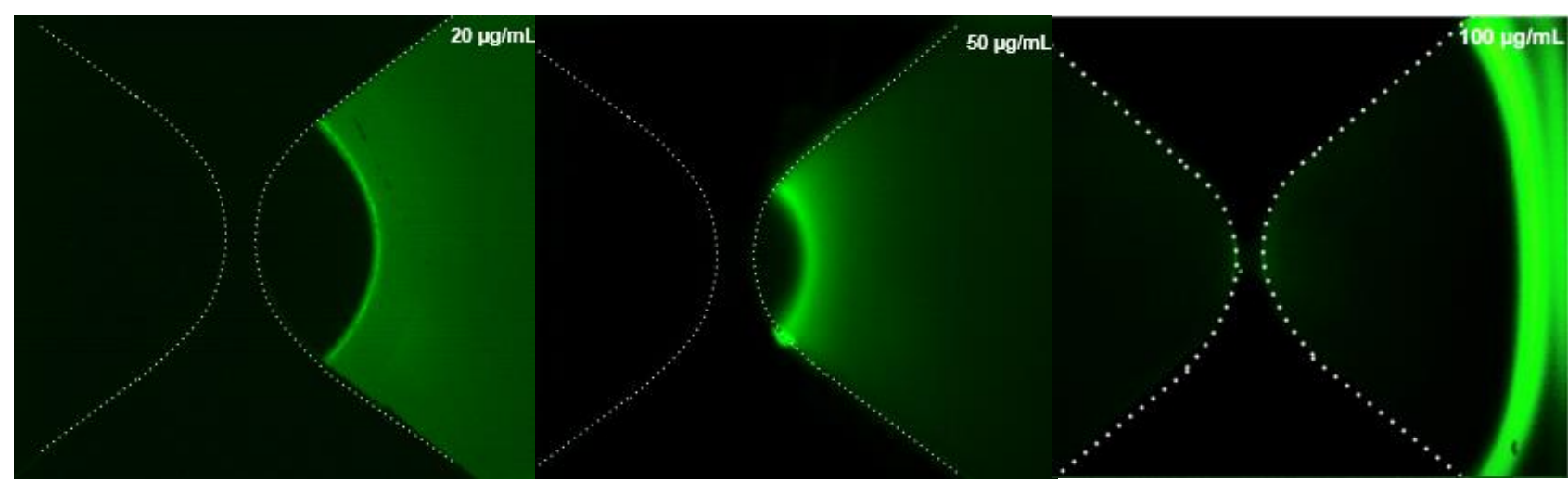

(d)

(e)

(f) 


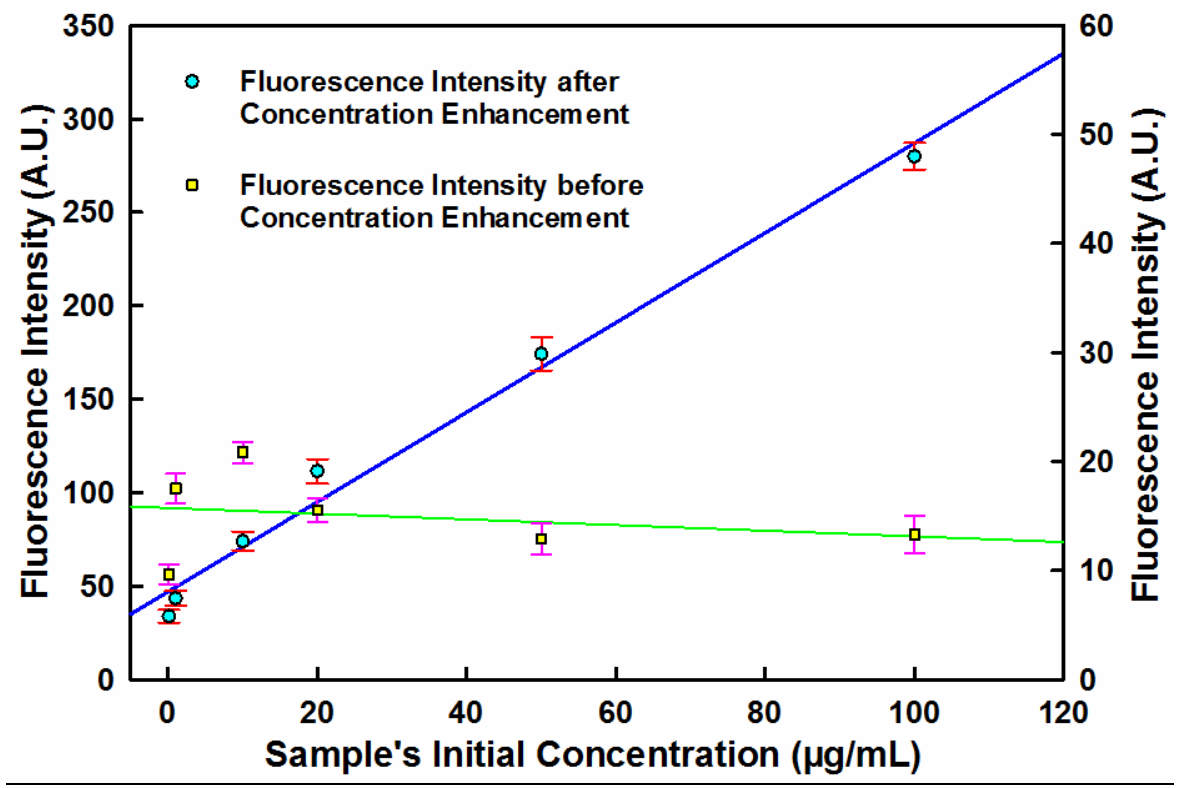

(g)

Figure 5-6. Representative FITC-BSA concentration results under the same electric field condition $\left(\mathrm{V}_{P P}=15 \mathrm{~V}\right.$, frequency $\left.=10 \mathrm{~Hz}, \mathrm{~V}_{D C}=2 \mathrm{~V}\right)$ with different initial solution concentrations. (a) (f) Fluorescence images show the results after the electric field applied for 120 seconds using an initial solution concentration of $100 \mathrm{ng} / \mathrm{mL}, 1 \mu \mathrm{g} / \mathrm{mL}, 10 \mu \mathrm{g} / \mathrm{mL}, 20 \mu \mathrm{g} / \mathrm{mL}, 50 \mu \mathrm{g} / \mathrm{mL}$ and $100 \mu \mathrm{g} / \mathrm{mL}$, respectively. (g) Relations between the average fluorescence intensities within the concentration region and sample's initial concentrations before and after the protein concentration process, respectively. The data is obtained from the results shown in (a) (f) using the same measurement method illustrated in Figure 5-2 (d). 


\section{Chapter 6 Conclusions and Future Work}

Microfluidic systems are becoming a powerful alternative used for carrying out a wide range of tasks including biological analysis and biochemical detection. However, due to the fact that the presence of the interested biomarker applied for detection is always far beyond the lower limits of detection technologies, it is significant to incorporate sample preparation modules. In order to achieve rapid and effective sample enrichment, DEP based technologies have been widely used to deal with this issue. Recently, there is a growing need for iDEP microfluidic devices with low fabrication complexity and cost, and the possibility for easy integration with existing devices for potential bioanalysis applications.

In this research work, successful protein concentration was demonstrated by a combination effect of electrokinetics and insulating-based dielectrophoresis under DC biased AC electric fields in a PDMS-based microfluidic device. The iDEP device we designed with a geometry of sub-micron sized constricted channel was conveniently fabricated by DNA molecular combing and replica molding, and the major fabrication processes only require equipment for standard photolithography and soft lithography techniques to create the constricted channel embedded within a microfluidic channel. The fabrication method applied is simple and low-cost without relying on e-beam lithography to fabricate the sub-micron channel.

Through theoretical analysis and experimental tests, the mechanism of protein concentration based on the balance of negative dielectrophoretic force and DC electrokinetic forces was well demonstrated. When a low DC bias (1-2V) and low-frequency AC electric field with moderate amplitude ( $\left.15 \mathrm{~V}_{\mathrm{PP}}\right)$ was applied, the embedded sub-micron size constricted channel was able to significantly enhance the local electric field, which proved to be strong enough to concentrate the protein molecules in very short time. This makes the device more useful by avoiding applying a 
very high frequency electric field. Additionally, the effects of applied electric field parameters (DC bias, AC amplitudes, AC frequencies) and suspending medium conductivities on the protein concentration responses (concentration bands focusing region and its intensities) were studied, demonstrating an optimal condition for protein concentration. Meanwhile, the measurement sensitivity for the samples with various initial concentrations was also tested to verify the applicability of the developed device under a wide range of sample conditions.

The current iDEP microfluidic device is basically designed for the sample concentration of a single biomarker (protein), but it will be further modified to obtain multiplexing capabilities. Moreover, future work will be conducted on the integration of the designed device with other micro/nano scale units developed for biomarker detection and measurement in order to create a portable complex microfluidic system that could provide great potential in practical point-of-care applications and allow generating more meaningful and conclusive information for clinical diagnosis. 


\section{Bibliography}

[1] C. Church. "Electrokinetic Manipulation of Particles and Cells in Microfluidic Devices", Clemson University, 2010.

[2] T. Nguyen, R. Pei, M. Stojanovic and Q. Lin. "Demonstration and Characterization of Biomolecular Enrichment on Microfluidic Aptamer-Functionalized Surfaces", Sens Actuators B Chem., vol.155, no. 1, pp. 58-66, 2011.

[3] J. C. McDonald, D. C. Duffy, J. R. Anderson, D. T. Chiu, H. K. Wu, O. J. A. Schueller, and G. M. Whitesides, "Fabrication of microfluidic systems in poly(dimethylsiloxane)", Electrophoresis, vol. 21, pp. 27-40, 2000.

[4] C. Zhang. "Investigation of Dielectrophoresis and Its Applications in Micro/nano Fluidics, Electronics and Fabrications", RMIT University, Melbourne, 2010.

[5] R. Yang. "On Chip Preconcentration and Labeling of Protein Biomarkers Using Monolithic Columns, Device Fabrication, Optimization, and Automation”, Brigham Young University, 2014.

[6] A. Nakano, F. Camacho-Alanis and A. Ros. "Insulator-based dielectrophoresis with $\beta$ galactosidase in nanostructured devices", Analyst, vol. 140, pp. 860-868, 2015.

[7] M. Viefhues, R. Eichhorn, E. Fredrich, J. Regtmeier, D. Anselmetti. "Continuous and reversible mixing or demixing of nanoparticles by dielectrophoresis", Lab Chip, vol. 12, pp. 485-494, 2012.

[8] V. Polaskova, A. Kapur, A. Khan, M.P. Molloy, M.S. Baker. "High-abundance protein depletion: comparison of methods for human plasma biomarker discovery", Electrophoresis, vol. 31, pp. 471-482, 2010.

[9] Y. Yang, C. Li, K. H. Lee, H. G. Craighead. "Coupling on-Chip Solid-Phase Extraction to Electrospray Mass Spectrometry through Integrated Electrospray Tip", Electrophoresis, vol. 26, pp. 3622-3630, 2005.

[10] Y. Demircan, A. Koyuncuoglu, M. Erdem, E. Ozgur, U. Gunduz and H. Kulah. "Labelfree detection of multidrug resistance in K562 cells through isolated 3D-electrode dielectrophoresis", Electrophoresis, vol. 36, pp. 1149-1157, 2015.

[11] Tay F E H, Yu L, Pang A J and Iliescu C. Electrochimica Acta, vol. 52, pp. 2862-68, 2007.

[12] P. V. Jones, S. J. R. Staton and M. A. Hayes. "Blood cell capture in a sawtooth dielectrophoretic microchannel", Anal. Bioanal. Chem., vol. 401, pp. 2103-2111, 2011.

[13] S.-W. Nam, S. H. Kim, J.-K. Park, and S. Park, "Dielectrophoresis in a slanted microchannel for separation of microparticles and bacteria", Journal of nanoscience and nanotechnology, vol. 13, no. 12, pp. 7993- 7997, 2013.

[14] P. Zellner, T. Shake, A. Sahari, B. Behkam and M. Agah. "Off-chip passivated-electrode, insulator-based dielectrophoresis (O $\pi \mathrm{DEP})$ ", Anal. Bioanal. Chem., vol. 405, pp. 6657-6666, 2013.

[15] T. Masuda, H. Maruyama, A. Honda and F. Arai. "Virus enrichment for single virus infection by using 3D insulator based dielectrophoresis", PLOS One, vol. 9, e94083, 2014.

[16] R. C. Gallo-Villanueva, C. E. Rodriguez-Lopez, R. I. Diaz-de-la-Garza, C. ReyesBetanzo and B. Lapizco-Encinas. "DNA manipulation by means of insulator-based dielectrophoresis employing direct current electric fields", Electrophoresis, vol. 30, pp. 4195-4205, 2009. 
[17] C.F. Chou, J. O. Tegenfeldt, O. Bakajin, S. S. Chan, E. C. Cox, N. Darnton, T. Duke, and R. H. Austin. "Electrodeless Dielectrophoresis of Single- and Double-Stranded DNA", Biophys., vol. 83, pp. 2170-2179, 2002.

[18] L. Lesser-Rojas, P. Ebbinghaus, G. Vasan, M. L. Chu, A. Erbe and C. F. Chou. "LowCopy Number Protein Detection by Electrode Nanogap-Enabled Dielectrophoretic Trapping for Surface-Enhanced Raman Spectroscopy and Electronic Measurements", Nano Lett., vol.14, pp. 2242-2250, 2014.

[19] M. Javanmard, S. Emaminejad, R. W. Dutton and R.W. Davis. "Use of Negative Dielectrophoresis for Selective Elution of Protein-bound Particles", Anal Chem., vol.84, pp. 1432-38, 2012.

[20] L. Gan. "Insulator-Based Dielectrophoretic Manipulation of DNA in a Microfluidic Device", Arizona State University, 2015.

[21] C. Gouvea. "Biosensors for health applications". Biosensors for Health, Environment and Biosecurity, P. A. Serra, Ed. InTech, 2011.

[22] D. Juncker, H. Schmid, U. Drechsler, H. Wolf, M. Wolf, B. Michel, N. de Rooij, and E. Delamarche, "Autonomous Microfluidic Capillary System," Anal. Chem., vol. 74, no. 24, pp. 6139-6144, 2002.

[23] N. Swami, C. F. Chou, V. Ramamurthy and V. Chaurey. "Enhancing DNA hybridization kinetics through constriction-based dielectrophoresis", Lab Chip, vol. 9, pp.3212-3220, 2009.

[24] L. Wu, Y. Lanry and K. M. Lim. "Dielectrophoretic capture voltage spectrum for measurement of dielectric properties and separation of cancer cells", Biomicrofluidics, vol. 6, $014113,2012$.

[25] C. H. Chiou, J. C. Pan, L. J. Chien, Y. Y. Lin, and J. L. Lin. "Characterization of Microparticle Separation Utilizing Electrokinesis within an Electrodeless Dielectrophoresis Chip", Sensors, vol. 13, pp. 2763-2776, 2013.

[26] B.H. Lapizco-Encinas, R.V. Davalos, B.A. Simmons, E.B. Cummings, Y.Fintschenko. "An insulator-based (electrodeless) dielectrophoretic concentrator for microbes in water", $J$. Microbiol. Methods, vol. 62, pp. 317-326, 2005.

[27] B. Bilenberg, S. Jacobsen, M.S. Schmidt, L.H.D. Skjolding, P. Shi, P. Bøggild, J.O. Tegenfeldt, A. Kristensen. "High resolution 100 kV electron beam lithography in SU-8", Microelectronic Engineering, vol. 83, pp. 1609-1612, 2006.

[28] L. C. Campbell, M. J. Wilkinson, A. Manz, P. Camilleri and C. J. Humphreys. "Electrophoretic manipulation of single DNA molecules in nanofabricated capillaries", Lab Chip, VO. 4, pp. 225-229, 2004.

[29] L. Ying, S. S. White, A. Bruckbauer, L. Meadows, Y. Korchev and D. Klenerman. " Frequency and Voltage Dependence of the Dielectrophoretic Trapping of Short Lengths of DNA and dCTP in a Nanopipette", Biophys. J. vol. 86, pp. 1018-1027, 2004.

[30] P. E. Boukany, A. Morss, W. C. Liao, B. Henslee, H. Jung, X. Zhang, B. Yu, X. Wang, Y. Wu, L. Li, K. Gao, X. Hu, X. Zhao, O. Hemminger, W. Lu, G. P. Lafyatis, and L. J. Lee, "Nanochannel electroporation delivers precise amounts of biomolecules into living cells", Nat. Nanotechnol. vol.6, no.11, pp. 747-754, 2011.

[31] P. Boukany, Y. Wu, X. Zhao, K. J. Kwak, P. J. Glazer, K. Leong and L. Lee." Nonendocytic delivery of lipoplex nanoparticles into living cells using nanochannel electroporation", Adv Healthc Mater, vol. 3, pp. 682-689, 2014. 
[32] J. Guan, P. E. Boukany, O. Hemminger, N. R. Chiou, W. Zha, M. Cavanaugh, and L. J. Lee. "Large laterally ordered nanochannel arrays from DNA combing and imprinting", $A d v$. Mater., vol. 22, no.36, pp. 3997-4001, 2010.

[33] J. Guan, L. J. Lee. "Generating highly ordered DNA nanostrand arrays", Proc Natl Acad Sci USA, vol.102, no. 51, pp. 18321-18325, 2005.

[34] A. Pohl. "The motion and precipitation of suspensoids in divergent electric fields", Journal of Applied Physics, vol. 22, pp. 869-871, 1951.

[35] R. Pethig. "Dielectrophoresis: Using inhomogeneous ac electrical fields to separate and manipulate cells", Critical Reviews in Biotechnology, vol. 16, no. 4, pp. 331-348, 1996.

[36] M. Washizu, S. Suzuki, O. Kurosawa, T. Nishizaka, T. Shinohara. "Molecular dielectrophoresis of biopolymers", IEEE Trans. Ind. Appl., vol. 30, pp. 835-843, 1994.

[37] D.J. Bakewell, M.P. Hughes, J.J. Milner, H. Morgan. "Dielectrophoretic manipulation of avidin and DNA", 20th International Conference of the IEEE in Medicine and Biology Society, vol. 20, no. 2, pp. 1079, 1998.

[38] M. P. Hughes. Nanoelectromechanics in Engineering and Biology (1st ed.). Boca Raton: CRC Press, 2002.

[39] B.H. Lapizco-Encinas, M. Rito-Palomares. "Dielectrophoresis for the manipulation of nanobioparticles", Electrophoresis, vol. 28, pp. 4521-4538, 2007.

[40] A. Nakano. "Protein Dielectrophoresis Using Insulator-based Microfluidic Platforms", Arizona State University, 2014.

[41] T. J. Shake. "Embedded Passivated-electrode Insulator-based Dielectrophoresis

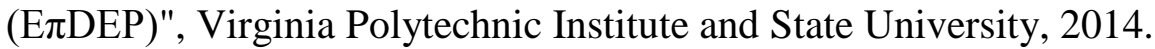

[42] C. Chung, I. Cheng, H. Chen, H. Kan, W. Yang and H. Chang. "Screening of Antibiotic Susceptibility to beta-Lactam-Induced Elongation of Gram-Negative Bacteria Based on Dielectrophoresis", Analytical Chemistry, vol. 84, no. 7, pp. 3347-3354, 2012.

[43] L. Zheng, J. Brody and P. Burke. "Electronic manipulation of DNA, proteins, and nanoparticles for potential circuit assembly", Biosensors \& bioelectronics, vol. 20, no. 3, pp. 606-619, 2004.

[44] L. Zheng, S. Li, J. Brody and P. Burke. "Manipulating nanoparticles in solution with electrically contacted nanotubes using dielectrophoresis", Langmuir, vol. 20, no. 20, pp. 8612-8619, 2004.

[45] V. Linko, S. Paasonen, A. Kuzyk, P. Torma and J.J. Toppari. "Characterization of the Conductance Mechanisms of DNA Origami by AC Impedance Spectroscopy", Small, vol. 5, no. 21, pp. 2382-2386, 2009.

[46] C. Schaefer, D.P.Kern and M. Fleischer. "Capturing molecules with plasmonic nanotips in microfluidic channels by dielectrophoresis", Lab Chip, vol. 15, no. 4, pp. 1066-1071, 2015.

[47] Y. Kim, X. Ren, J.W.Kim and H. Noh. "Direct inkjet printing of micro-scale silver electrodes on polydimethylsiloxane (PDMS) microchip", Journal of Micromechanics and Microengineering, vol. 24, no. 11, pp. 115010, 2014.

[48] R. Martinez-Duarte, F. Camacho-Alanis, P. Renaud and A. Ros. "Dielectrophoresis of lambda-DNA using 3D carbon electrodes", Electrophoresis, vol. 34, no. 7, pp. 1113-1122, 2013.

[49] C. Zhang, K. Khoshmanesh, A. Mitchell, and K. Kalantar-zadeh. "Dielectrophoresis for manipulation of micro/nano particles in microfluidic systems", Anal. Bioanal. Chem., vol. 396, pp. 401-420, 2009. 
[50] J. Regtmeier, R. Eichhorn, M. Viefhues, L. Ogunovic, D. Anselmetti. "Electrodeless dielectrophoresis for bioanalysis: theory, devices and applications", Electrophoresis, vol. 32, pp. 2253-2273, 2011.

[51] H. Shafiee, J.L. Caldwell, M.B. Sano, and R.V. Davalos. "Contactless dielectrophoresis: a new technique for cell manipulation", Biomedical Microdevices, vol. 11, no. 5, pp. 997 1006, 2009.

[52] L. Gan, T.C. Chao, F. Camacho-Alanis and A. Ros. "Six-helix bundle and triangle DNA origami insulator-based dielectrophoresis", Anal. Chem., vol. 85, no. 23, pp. 11427-11434, 2013.

[53] F. Camacho-Alanis, L. Gan and A. Ros. "Transitioning streaming to trapping in DC insulator-based dielectrophoresis for biomolecules", Sensors and Actuators B-Chemical, vol. 173, pp. 668-675, 2012.

[54] E. B. Cummings and A. K. Singh. "Dielectrophoresis in Microchips Containing Arrays of Insulating Posts: Theoretical and Experimental Results", Anal. Chem., vol. 75, 4724-4731, 2003.

[55] J. Zhu, X. Xuan. "Dielectrophoretic focusing of particles in a microchannel constriction using DC-biased AC electric fields", Electrophoresis, vol. 30, pp.2668-2675, 2009.

[56] B. H. Lapizco-Encinas, B.A. Simmons, E.B. Cummings, Y. Fintschenko. "Insulatorbased dielectrophoresis for the selective concentration and separation of live bacteria in water", Electrophoresis, vol. 25, pp.1695-1704, 2004.

[57] P. Sabounchi, A.M. Morales, P. Ponce, L.P. Lee, B.A. Simmons, R.V. Davalos. "Sample concentration and impedance detection on a microfluidic polymer chip", Biomed microdevices, vol. 10, no. 5, pp. 661-670, 2008.

[58] S. Srivastava, A. Gencoglu, A. Minerick. "DC insulator dielectrophoretic applications in microdevice technology: a review", Anal. Bioanal. Chem., vol. 399, pp. 301-321, 2011.

[59] S. K. Srivastava, J. L. Baylon-Cardiel, B. H. Lapizco-Encinas and A. R. Minerick. " A continuous DC-insulator dielectrophoretic sorter of microparticles", J. Chromatogr. A, vol. 1218, pp. 1780-1789, 2011.

[60] Y. Kang, D. Li, S. Kalams and J. Eid. "DC-Dielectrophoretic separation of biological cells by size", Biomed. Microdevices, vol. 10, pp. 243-249, 2008.

[61] K. H. Kang, Y. Kang, X. Xuan and D. Li. " Continuous separation of microparticles by size with Direct current-dielectrophoresis", Electrophoresis, vol. 27, pp. 694-702, 2006.

[62] A. Gencoglu, F. Camacho-Alanis, V. T. Nguyen, A. Nakano, A. Ros and A. R. Minerick. "Quantification of $\mathrm{pH}$ gradients and implications in insulator-based dielectrophoresis of biomolecules", Electrophoresis, vol. 32, pp. 2436-2447, 2011.

[63] Y.H. Su, M. Tsegaye, W. Varhue, K.-T. Liao, L. S. Abebe, J. A. Smith, R. L. Guerrant and N. S. Swami. "Quantitative dielectrophoretic tracking for characterization and separation of persistent subpopulations of Cryptosporidium parvum" Analyst, vol. 139, pp. 66-73, 2014.

[64] P. V. Jones, S. H. Hilton, P. E. Davis, R. Yanashima, R. McLemore, A. McLaren and M. A. Hayes. "Biophysical separation of Staphylococcus epidermidis strains based on antibiotic resistance" Analyst, vol. 140, pp. 5152-5161, 2015.

[65] K. P. Chen, J. R. Pacheco, M. A. Hayes and S. J. R. Staton. "Insulator-based dielectrophoretic separation of small particles in a sawtooth channel", Electrophoresis, vol. 30, pp. 1441-1448, 2009. 
[66] S. J. R. Staton, K. P. Chen, T. J. Taylor, J. R. Pacheco and M. A. Hayes. "Characterization of particle capture in a sawtooth patterned insulating electrokinetic microfluidic device", Electrophoresis, vol. 31, pp. 3634-3641, 2010.

[67] A. LaLonde, A. Gencoglu, M. F. Romero-Creel, K. S. Koppula and B. H. LapizcoEncinas. "Effect of insulating posts geometry on particle manipulation in insulator based dielectrophoretic devices", J. Chromatogr. A, vol. 1344, pp. 99-108, 2014.

[68] E. B. Cummings. "Streaming dielectrophoresis for continuous-flow microfluidic devices", IEEE Eng. Med. Biol. Mag., vol. 22, pp. 75-84, 2003.

[69] M. Viefhues, J. Regtmeier and D. Anselmetti. "Fast and continuous-flow separation of DNA-complexes and topological DNA variants in microfluidic chip format", Analyst, vol. 138, no. 1, pp. 186-196, 2013.

[70] B.H. Lapizco-Encinas, S. Ozuna-Chacón and M. Rito-Palomares. "Protein manipulation with insulator-based dielectrophoresis and direct current electric fields", J. Chromatogr. A, vol. 1206, pp. 45-51, 2008.

[71] S.J. Staton, P.V. Jones, G. Ku, S.D. Gilman, I. Kheterpal and M.A. Hayes. "Manipulation and capture of $A \beta$ amyloid fibrils and monomers by DC insulator gradient dielectrophoresis (DC-iGDEP)", Analyst, vol. 137, no.14, pp. 3227-3229, 2012.

[72] S. Hardt and F. Schönfeld. "Microfluidic Technologies for Miniaturized Analysis Systems", Springer US, New York, 2007.

[73] R.W. Clarke, S.S. White, D. Zhou, L. Ying and D. Klenerman. "Trapping of proteins under physiological conditions in a nanopipette", Angewandte. Chemie International Edition. vol. 44, no. 24, pp. 3747-3750, 2005.

[74] R.W. Clarke, J.D. Piper, L. Ying and D. Klenerman. "Surface conductivity of biological macromolecules measured by nanopipette dielectrophoresis", Physical Review Letters, vol. 98, no.19, pp. 198102(1)-198102(4), 2007.

[75] C. Chou, F. Zenhausern. "Electrodeless dielectrophoresis for micro total analysis systems", IEEE Eng. Med. Biol. Mag., vol. 22, pp. 62-67, 2003.

[76] K.T. Liao, C.F. Chou. "Nanoscale molecular traps and dams for ultrafast protein enrichment in high-conductivity buffers", Journal of the American Chemical Society, vol. 134, pp. 8742-8745, 2012.

[77] K.T. Liao, M. Tsegaye, V. Chaurey, C.F. Chou and N. Swami. "Nano-constriction device for rapid protein preconcentration in physiological media through a balance of electrokinetic forces", Electrophoresis, vol. 33, pp. 1958-1966, 2012.

[78] A. Pohl. "Dielectrophoresis", Cambridge University Press, Cambridge, 1978.

[79] C. Zhang, K. Khoshmanesh, A. Mitchell, and K. Kalantar-zadeh. "Dielectrophoresis for manipulation of micro/nano particles in microfluidic systems", Anal. Bioanal. Chem., vol. 396, pp. 401-420, 2009.

[80] R. F. Probstein. "Physicochemical Hydrodynamics An Introduction, 2nd edn", John Wiley \& Sons, Inc., Hoboken, New Jersey, 2003.

[81] M. von Smoluchowski. "Elektrische endosmose und stromungsstrome. In Handbuch der Elektrizitat und des Magnetimus (Vol. 2)". J. A. Barth, Leipzig, 1914.

[82] J. Lyklema. "Fundamentals of Interface and Colloid Science (Vols. 1-2)", MA: Academic Press, Waltham, 1995.

[83] M. von Smoluchowski. "Contribution à la théorie de l'endosmose électrique et de quelques phénomènes corrélatifs", Bull. Int. Acad. Sci. Cracovie, pp. 182-189, 1903. 
[84] A. Gencoglu, D.N. Olney, A. LaLonde, K.S. Koppula and B.H. Lapizco-Encinas. "Particle Manipulation in Insulator Based Dielectrophoretic Devices", J. Nanotechnol. Eng. Med., vol.4, pp. 021002, 2013.

[85] P. Zhang, Y. Liu. "DC biased low-frequency insulating constriction dielectrophoresis for protein biomolecules concentration", Biofabrication, vol. 9, pp. 045003, 2017.

[86] L. Gervais, N. de Rooij, and E. Delamarche. "Microfluidic Chips for Point-of-Care Immunodiagnostics”, Adv. Mater., vol. 23, no. 24, pp. H151-H176, Jun. 2011.

[87] L. Ristić. "Sensor technology and devices", Artech House, 1994.

[88] D.J. Harrison, A. Manz, Z. Fan, H. Luedi. H.M. Widmer. "Capillary electrophoresis and sample injection systems integrated on a planar glass chip", Anal. Chem., vol. 64, pp. 19261932, 1992.

[89] V. Betancur. "An integrated lateral flow device for flow control and blood separation", West Virginia University, 2016.

[90] T. Vestad, D. W. M. Marr, and J. Oakey. "Flow control for capillary-pumped microfluidic systems". J. Micromechanics Microengineering, vol. 14, no. 11, pp. 1503-1506, 2004.

[91] J. Guan, B. Yu, L.J. Lee. "Forming highly ordered arrays of functionalized polymer nanowires by dewetting on micropillars", Adv Mater, vol. 19, pp. 1212-1217, 2006.

[92] C. H. Lin, J. Guan, S. W. Chau, S. C. Chen and L. J. Lee. "Patterning nanowire and micro-/nanoparticle array on micropillar-structured surface: Experiment and modeling", Bio microfluidics, vol.4, pp. 034103-1-034103-15, 2010.

[93] V. Chaurey, A. Rohani, Y.-H. Su, K.-T. Liao, C.-F. Chou, N.S. Swami. "Scaling down constriction-based (electrodeless) dielectrophoresis devices for trapping nanoscal bioparticles in physiological media of high-conductivity", Electrophoresis, vol. 34, pp. 1097-1104, 2013.

[94] V. Chaurey, C. Polanco, C.F. Chou, N.S. Swami. "Floating-electrode enhanced constriction dielectrophoresis for biomolecular trapping in physiological media of high conductivity", Biomicrofluidics, vol.6, pp. 012806, 2012.

[95] T. Tadros. "Dispersion of Powders in Liquids and Stabilization of Suspensions", Wiley-VCH, Weinheim, 2012. 